Wetting fronts in unsaturated porous media: The combined case of hysteresis and dynamic capillary pressure

Non Peer-reviewed author version

MITRA, Koondanibha \& Van Duijn, Cornelis Johannes (2019) Wetting fronts in unsaturated porous media: The combined case of hysteresis and dynamic capillary pressure. In: NONLINEAR ANALYSIS-REAL WORLD APPLICATIONS, 50, p. 316-341.

DOI: 10.1016/j.nonrwa.2019.05.005

Handle: http://hdl.handle.net/1942/29034 


\section{UHASSELT}

\section{Wetting fronts in unsaturated porous media: the combined case of hysteresis and dynamic capillary}

Koondanibha Mitra and Hans van Duijn

UHasselt Computational Mathematics Preprint Nr. UP-18-03

June 15, 2018 


\title{
Wetting fronts in unsaturated porous media: the combined case of hysteresis and dynamic capillary pressure
}

\author{
K. Mitra*1,2 and C.J. van Duijn ${ }^{3,4}$ \\ ${ }^{1}$ Eindhoven University of Technology, Department of Mathematics and Computer Science \\ ${ }^{2}$ Eindhoven University of Technology, Department of Mechanical Engineering \\ ${ }^{3}$ Utrecht University, Department of Earth Sciences
}

\begin{abstract}
This paper extends the work of [14] where travelling wave solutions for wetting fronts were considered under the presence of only capillary hysteresis effect and only dynamic capillary effect. In this work, we investigate how the gravity driven wetting fronts behave while moving through long vertical homogeneous porous columns, under the combined effect of capillary hysteresis and dynamic capillarity. It is shown that the developed saturation profiles will exhibit non-monotone behaviour if certain parametric conditions are satisfied. The characteristics of the profiles are explained in detail for all the cases. Moreover, parametric conditions that inhibit the fronts from reaching full saturation are laid out. The analysis agrees well with experimental observations. Finally, numerical results are shown that confirm all the theoretical predictions.
\end{abstract}

\section{Introduction}

In the recent paper [14], we considered wetting fronts in the form of travelling wave solutions of the one-dimensional Richards equation with either capillary hysteresis or dynamic capillarity. In the scaled setting, the involved equations are given by the fluid transport equation,

$$
\partial_{t} S+\partial_{x}\left(k(S)\left(\partial_{x} u+1\right)\right)=0
$$

\footnotetext{
*Corresponding author: email: k.mitra@tue.nl
} 
combined with either capillary hystersis

$$
u \in \frac{P_{d}(S)+P_{i}(S)}{2}-\frac{P_{d}(S)-P_{i}(S)}{2} \operatorname{sign}\left(\partial_{t} S\right),
$$

or dynamic capillarity

$$
u=P_{c}(S)-\tau f(S) \partial_{t} S .
$$

Here $\operatorname{sign}(\cdot)$ is the multivalued signum function

$$
\operatorname{sign}(\zeta)= \begin{cases}1 & \text { for } \zeta>0 \\ {[-1,1]} & \text { for } \zeta=0 \\ -1 & \text { for } \zeta<0\end{cases}
$$

Thus in [14] we studied the combinations (1.1), (1.2a) and (1.1), (1.2b). It is the purpose of the present paper to study wetting fronts in the form of travelling waves for the combined case of capillary hysteresis and dynamic capillarity. Then (1.2a) and (1.2b) are written as the single expression

$$
u \in \frac{P_{d}(S)+P_{i}(S)}{2}-\frac{P_{d}(S)-P_{i}(S)}{2} \operatorname{sign}\left(\partial_{t} S\right)-\tau f(S) \partial_{t} S .
$$

In the above equations, $S \in[0,1]$ is the water saturation and $u$ the water pressure. The function $k(S)$ denotes the relative permeability and $P_{d}(S)$ and $P_{i}(S)$ are respectively, the primary (main) drainage and imbibition capillary pressure functions. In the absence of hysteresis they are the same and are denoted by $P_{c}(S)$. Further, $\tau$ is the dynamic capillary coefficient and $f(S)$ the dynamic capillary function.

As in [14] we assume

(A.1) $k \in \mathcal{C}^{1}([0,1]), k^{\prime}(S)>0$ for $0<S \leq 1, k(0)=0, k(1)=1$ and $k$ is strictly convex in $[0,1]$.

(A.2) The capillary coefficient is positive, $\tau>0$. The capillary function $f \in \mathcal{C}^{1}([0,1))$ and $f(S)>0$ for $0<S<1$.

(A.3) The capillary pressure functions $P_{k}(k=i, d)$ satisfy $P_{k}:(0,1] \rightarrow[0, \infty), P_{k} \in$ $\mathcal{C}^{1}((0,1]), P_{k}(1)=0, P_{k}{ }^{\prime}(S)<0$ and $P_{i}(S)<P_{d}(S)$ for $0<S<1$.

In (A.1)-(A.3), and elsewhere in this paper, a prime denotes differentiation with respect to the argument.

The combined pressure equation (1.4) implies that if $\partial_{t} S>0$, then

$$
\tau f(S) \partial_{t} S=P_{i}(S)-u
$$

and if $\partial_{t} S<0$, then

$$
\tau f(S) \partial_{t} S=P_{d}(S)-u
$$


Using this observation, (1.4) can be rewritten as, see also [1],

$$
\partial_{t} S=\frac{1}{\tau f(S)} \begin{cases}P_{d}(S)-u & \text { when } u \geq P_{d}(S), \\ 0 & \text { when } P_{i}(S)<u<P_{d}(S), \\ P_{i}(S)-u & \text { when } u \leq P_{i}(S) .\end{cases}
$$

Hence the model based on (1.4), or on (1.2a), implies vertical scanning curves $\left(\partial_{t} S=0\right)$ when $P_{i}(S)<u<P_{d}(S)$. For this reason, hysteresis described by (1.4) is called play-type hysteresis $[1,15]$.

In this paper we consider, in addition to (1.4), an extension of play-type hysteresis by introducing non-vertical scanning curves. It is achieved by adapting (1.4) in the following way (for small $\varepsilon>0$ )

$$
u \in \frac{P_{d}(S)+P_{i}(S)}{2}-\frac{P_{d}(S)-P_{i}(S)}{2} \operatorname{sign}\left(\partial_{t} S+\varepsilon \partial_{t} u\right)-\tau f(S) \partial_{t} S .
$$

Such an extension was proposed in [6] and similar models were studied in $[1,17]$. In $[6]$ it is shown that the model is consistent if $\varepsilon$ satisfies certain upper bounds. The specific bounds are stated explicitly in Section 3.

As in (1.5), $\partial_{t} S$ can be made explicit from (1.6). The calculations are given in the Appendix. The result is

$$
\partial_{t} S=\mathcal{A}\left(S, u, \partial_{t} u\right):= \begin{cases}\frac{P_{d}(S)-u}{\tau f(S)} & \text { when } u \geq P_{d}(S)+\varepsilon \tau f(S) \partial_{t} u, \\ -\varepsilon \partial_{t} u & \text { when } P_{i}(S)+\varepsilon \tau f(S) \partial_{t} u<u<P_{d}(S)+\varepsilon \tau f(S) \partial_{t} u, \\ \frac{P_{i}(S)-u}{\tau f(S)} & \text { when } u \leq P_{i}(S)+\varepsilon \tau f(S) \partial_{t} u .\end{cases}
$$

Letting $\varepsilon \rightarrow 0$ in this expression yields the play-type expression (1.5) and letting $\tau \rightarrow 0$ gives

$$
\partial_{t}(S+\varepsilon u)=0 \text { when } P_{i}(S)<u<P_{d}(S),
$$

i.e. scanning curves in the form of straight lines with inclination $-\frac{1}{\varepsilon}[6]$.

Using (1.5) to eliminate $\partial_{t} S$ from (1.1), yields an elliptic equation for $u$. Likewise, using (1.7) in (1.1) gives an elliptic-parabolic equation for which an initial pressure is required. This is natural for extended play-type problems, as was shown in [6] where we studied the case of horizontal redistribution.

Assumption (A.2) includes the possibility of the capillary function $f(S)$ having a singular behaviour at $S=1$. The behaviour of $f(S)$, as observed from experiments [3], suggests that this should indeed be taken into consideration. Therefore we distinguish, as in [14], between the classes 

A. $f \in L^{1}(0,1)$
B. $f \notin L^{1}(0,1)$

We show in Section 2 that $f \in L^{1}(0,1)$ is in essence equivalent to the case where $f(S)=$ constant $=1$.

It is known that the dynamic term in (1.2b) may cause overshoot or oscillations in solutions of (1.1), (1.2b). This has been observed both analytically (e.g. $[4,7,9,14])$ and numerically (e.g $[11,12,17])$. In mathematical terms, equations of the type $(1.1),(1.2 \mathrm{~b})$ do not admit a maximum principle. Moreover, the stronger the dynamic term in $(1.2 \mathrm{~b})$, the larger the overshoot/oscillations. To prevent the saturation to extend its physical maximal value $S=1$, the capillary pressure was extended as a graph in [14]. For the hysteretic case (1.2a), this means that both $P_{i}$ and $P_{d}$ are extended to be set-valued at $S=1$ :

$$
P_{k}(S)= \begin{cases}P_{k}(S)(\text { as in }(\text { A. } 3)) & \text { when } 0<S<1, \\ (-\infty, 0] & \text { when } S=1,\end{cases}
$$

for $k=i, d$. Definition (1.8) allows us to deal with large values of $\tau$, causing the porous medium to be saturated $(S=1)$ in parts of the domain. The set-valued pressure at $S=1$ acts as a natural saturation cut-off.

This paper is organized as follows. In Section 2 travelling waves describing wetting fronts are introduced for the model based on (1.1), (1.4) and (1.8). The main emphasis, Section 2.1, is on the case $f(S)=1$. Special attention is given to the role of the parameter $\tau$ in relation to extension (1.8). Generalizations to non-constant $f$ are given in Section 2.2 ( $f$ from class A) and in Section 2.3 ( $f$ from class B). In Section 3 travelling waves based on the extended expression (1.6) are treated, making the role of the hysteretic parameter $\varepsilon$ explicit. Section 4 shows computational results for the models (1.4) and (1.6). We present the conclusions in Section 5.

\section{Travelling waves with combined pressure (1.4)}

As in [14], we seek travelling wave solutions of the form

$$
S(x, t)=S(\zeta), \quad u(x, t)=u(\zeta) \text { and } \zeta=c t-x,
$$

where $-\infty<\zeta<\infty$ and

$$
\lim _{\zeta \rightarrow-\infty} S(\zeta)=S_{B}, \quad \lim _{\zeta \rightarrow \infty} S(\zeta)=S_{T}
$$

Here $S_{B}$ corresponds to the 'initial' saturation and $S_{T}$ to the 'injected' saturation. In the physical context of wetting fronts we choose

$$
0<S_{B}<S_{T} \leq 1
$$


Due to the convexity of $k(S)$, this choice is necessary for mathematical reasons as well $[13,14]$.

The boundary conditions for the pressure need special attention. For the moment we impose

$$
u^{\prime}( \pm \infty)=0 .
$$

Substituting (2.1) into (1.1) and (1.5) and applying conditions (2.2) and (2.4), details are given in [14], one finds for the wave speed $c$ the Rankine-Hugoniot expression

$$
c=\frac{k\left(S_{T}\right)-k\left(S_{B}\right)}{S_{T}-S_{B}}
$$

and for $S$ and $u$ the first order equations

$$
\begin{aligned}
S^{\prime} & =\frac{1}{c \tau f(S)} \mathcal{F}(S, u), \\
u^{\prime} & =\mathcal{G}\left(S ; S_{B}, S_{T}\right) .
\end{aligned}
$$

Here

$$
\mathcal{F}(S, u)= \begin{cases}P_{d}(S)-u & \text { when } u>P_{d}(S), \\ 0 & \text { when } P_{i}(S) \leq u \leq P_{d}(S), \\ P_{i}(S)-u & \text { when } u<P_{i}(S),\end{cases}
$$

and

$$
\mathcal{G}\left(S ; S_{B}, S_{T}\right)=1-\frac{k\left(S_{B}\right)+c\left(S-S_{B}\right)}{k(S)} .
$$

These functions are sketched in Figure 1.
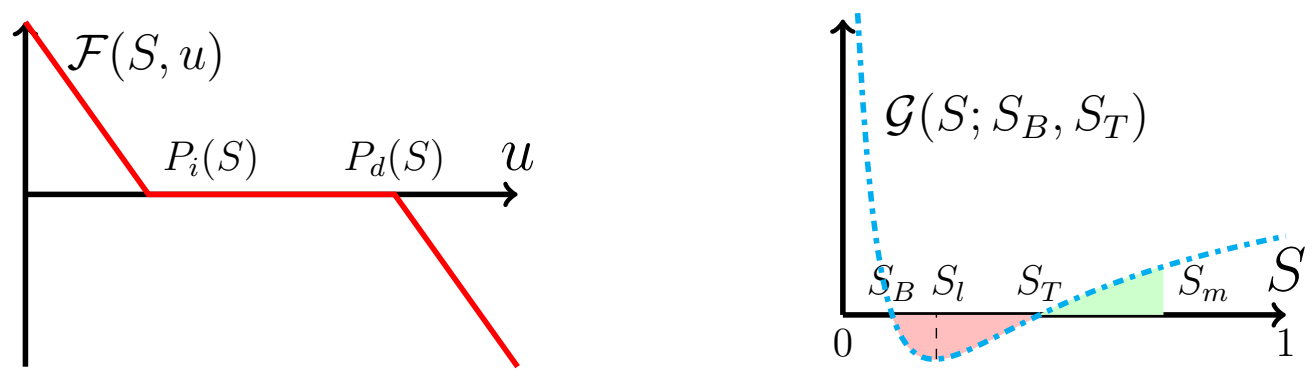

Figure 1: (left) Graph of $\mathcal{F}(S, u)$ for given $S \in(0,1)$, (right) Graph of $\mathcal{G}\left(S ; S_{B}, S_{T}\right)$ for given $0<S_{B}<S_{T}<1$, with $k(S)=S^{2}$. Here $S_{m}$ is the unique saturation value for which $\int_{S_{B}}^{S_{m}} \mathcal{G}\left(\varrho ; S_{B}, S_{T}\right) d \varrho=0$ and $S_{l} \in\left(S_{B}, S_{T}\right)$ is such that $\mathcal{G}\left(S_{l}\right)=\min _{S \in(0,1)} \mathcal{G}\left(S ; S_{B}, S_{T}\right)$.

Solutions of (2.6) belong to the strip $\{0<S \leq 1, u \in \mathbb{R}\}$. In this strip we introduce, 


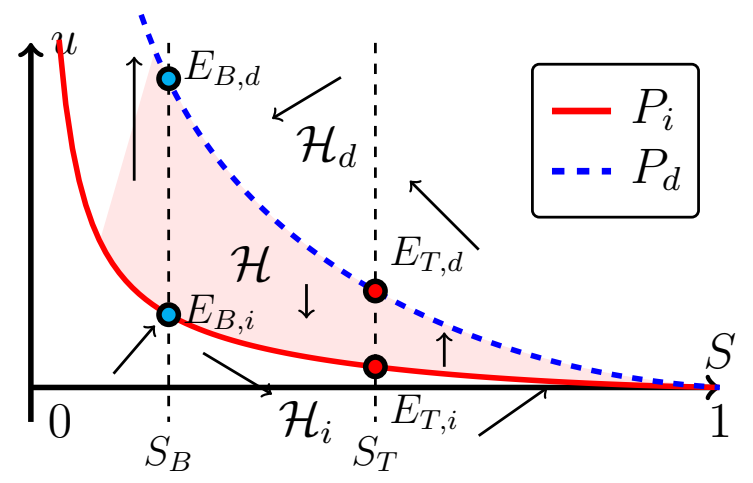

Figure 2: The sets (2.9) and points (2.10) in the strip $\{0<S \leq 1\}$. The arrows indicate the direction of the solution orbits (2.6)

for future reference, the sets.

$$
\begin{aligned}
& \mathcal{H}_{d}=\left\{u>P_{d}(S)\right\}, \\
& \mathcal{H}=\left\{P_{i}(S)<u<P_{d}(S)\right\}, \\
& \mathcal{H}_{i}=\left\{u<P_{i}(S)\right\},
\end{aligned}
$$

and the points $(k=i, d)$

$$
E_{B, k}=P_{k}\left(S_{B}\right), \quad E_{T, k}=P_{k}\left(S_{T}\right) .
$$

They are indicated in Figure 2, together with the direction of the solution orbits of (2.6).

The right hand side of (2.6) vanishes iff

$$
S \in\left\{S_{B}, S_{T}\right\} \text { and }(S, u) \in \mathcal{H}
$$

Hence all points of the closed segments $\overline{E_{B, i} E_{B, d}}$ and $\overline{E_{T, i} E_{T, d}}$ are equilibrium points of (2.6). Clearly, interior points cannot serve as start and/or end point of any trajectory. Only the end points $E_{B, k}$ and $E_{T, k}, k=i, d$, can play that role. We investigate the nature of these points later in this section.

With the capillary function $f$ satisfying (A.2), the function

$$
Y(S)=\int_{0}^{S} f(S) d S
$$

is well defined for $0 \leq S<1$ and $Y(1)<\infty$ iff $f \in L^{1}(0,1)$. Since $f>0$ in $(0,1)$, the inverse $S=S(Y)$ is uniquely determined by

$$
S=S(Y) \text { for } 0 \leq Y \leq Y(1) .
$$




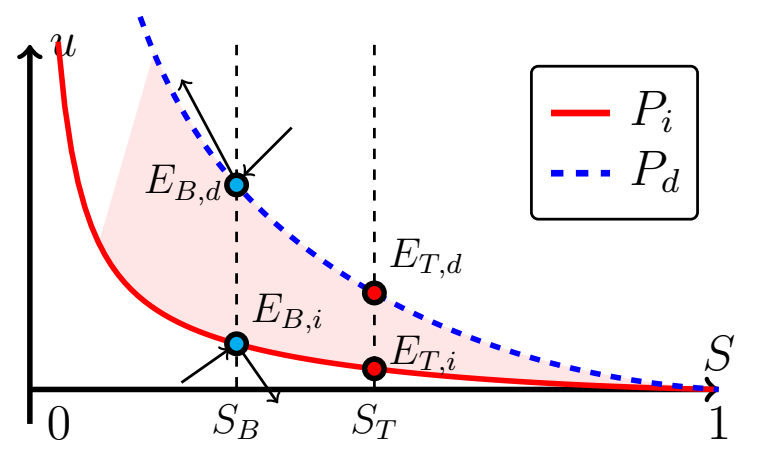

Figure 3: Eigenvectors of the saddles $E_{B, i}$ and $E_{B, d}$. The arrows indicate the direction of the corresponding trajectories for increasing $\zeta$.

Rewriting (2.6) in terms of $Y$ and $u$ gives

$$
\begin{aligned}
Y^{\prime} & =\frac{1}{c \tau} \tilde{\mathcal{F}}(Y, u), \\
u^{\prime} & =\tilde{\mathcal{G}}\left(Y ; Y_{B}, Y_{T}\right),
\end{aligned}
$$

where $\tilde{\mathcal{F}}(Y, u)=\mathcal{F}(S(Y), u)$ and $\tilde{\mathcal{G}}\left(Y ; Y_{B}, Y_{T}\right)=\mathcal{G}\left(S(Y) ; S_{B}, S_{T}\right)$ with $Y_{B, T}=\int_{0}^{S_{B, T}} f(S) d S$. Thus the transformed system only involves a stretching of the $S$-coordinate.

If $f \in L^{1}(0,1)$, solutions belong to the strip $\{0<Y \leq Y(1)\}$ and the transformed system (2.14) is qualitively similar to (2.6) with $f(S)=1$. Therefore the results for (2.6) with $f(S)=1$ can be translated directly to the case $f(S) \neq 1$ and $f \in L^{1}(0,1)$. If $f \notin L^{1}(0,1)$, solutions belong to the half space $\{Y>0\}$. This situation needs separate attention.

Remark 2.1. Since (2.6) is autonomous, its solutions are unique modulo a shift in $\zeta$. One usually fixes this by setting $S(0)=\frac{1}{2}\left(S_{B}+S_{T}\right)$.

\section{$2.1 \quad f(S)=1$}

Linearizing (2.6), by approaching $E_{B, i}$, from $\mathcal{H}_{i}$ and $E_{B, d}$ from $\mathcal{H}_{d}$, gives a positive and negative eigenvalue at these points. Hence $E_{B, i}$ and $E_{B, d}$ are saddles of which the direction of the eigenvectors is sketched in Figure 3

Similarly at the points $E_{T, i}$ and $E_{T, d}$ one finds for the eigenvalues (see also references $[4,9,12,14])$

$$
\lambda_{k}=\frac{P_{k}{ }^{\prime}\left(S_{T}\right)}{2 c \tau}\left[1 \pm \sqrt{1-\frac{\tau}{\tau_{c, k}}}\right]
$$

where

$$
\tau_{c, k}=\frac{\left(P_{k}^{\prime}\left(S_{T}\right)\right)^{2}}{4 c \mathcal{G}^{\prime}\left(S_{T} ; S_{B}, S_{T}\right)}>0 \quad(k=i, d) .
$$


Without loss of generality we may assume

$$
P_{d}^{\prime}\left(S_{T}\right)<P_{i}^{\prime}\left(S_{T}\right)<0
$$

implying

$$
\tau_{c, i}<\tau_{c, d}
$$

Then we have

- $\tau<\tau_{c, i} \Longrightarrow E_{T, i}$ and $E_{T, d}$ are sinks;

- $\tau_{c, i}<\tau<\tau_{c, d} \Longrightarrow E_{T, i}$ is stable spiral and $E_{T, d}$ is sink;

- $\tau>\tau_{c, d} \Longrightarrow E_{T, i}$ and $E_{T, d}$ are stable spirals.

As a consequence, if $\tau>\tau_{c, i}$, no orbit can reach $E_{T, i}$ along a fixed direction. This can only happen when $\tau<\tau_{c, i}$. The same holds at $E_{T, d}$ for $\tau>\tau_{c, d}$.

For a given $\tau>0$, the goal is to construct an orbit that runs from $S=S_{B}$ (and $\left.u=P_{i}\left(S_{B}\right)\right)$ as $\zeta \rightarrow-\infty$ to $S=S_{T}$ as $\zeta \rightarrow+\infty$. Inspection of the flow direction in Figure 2 gives that this can only be achieved by considering the orbit leaving $E_{B, i}$ in the direction of $\mathcal{H}_{i}$ for increasing $S$. As long as the orbit is in $\mathcal{H}_{i}$, we can use the results of [14] to describe its behaviour. Here we stay below $P_{i}$, whereas in [14] we stay below $P_{c}=P_{i}=P_{d}$. The behaviour of the orbit depends crucially on the value of $S_{T}$ with respect to $S_{B}$. To make this precise, we introduce as in [14] the expression

$$
\alpha\left(S_{B}, S_{T}\right)=\int_{S_{B}}^{1} \mathcal{G}\left(S ; S_{B}, S_{T}\right) d S
$$

where $\mathcal{G}$ is given by $(2.8)$.

Clearly, $\alpha\left(S_{B}, S_{T}=S_{B}\right)>0, \alpha\left(S_{B}, S_{T}=1\right)<0$ and $\alpha\left(S_{B}, \cdot\right)$ strictly decreases in $\left(S_{B}, 1\right)$; see Figure 4 for an impression. Hence for each $0<S_{B}<1$, there exists a unique $S_{T}=S_{T}^{*}\left(S_{B}\right)$ such that

$$
\alpha\left(S_{B}, S_{T}^{*}\left(S_{B}\right)\right)=0 \text { and }\left\{\begin{array}{l}
\alpha\left(S_{B}, S_{T}\right)>0 \text { if } S_{T}<S_{T}^{*}\left(S_{B}\right) \\
\alpha\left(S_{B}, S_{T}\right)<0 \text { if } S_{T}>S_{T}^{*}\left(S_{B}\right)
\end{array}\right.
$$

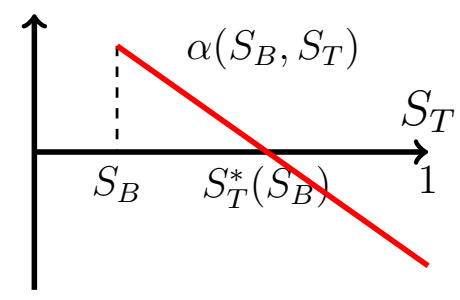

Figure 4: Sketch of expression (2.18) and location of $S_{T}^{*}\left(S_{B}\right)$. The plot shown is for $S_{B}=0.2$ and $k(S)=S^{2}$. 
Further, for $S_{T}<S_{T}^{*}\left(S_{B}\right)$, we define the saturation $S_{m} \in\left[S_{T}, 1\right]$ by

$$
\int_{S_{m}}^{1} \mathcal{G}\left(S ; S_{B}, S_{T}\right) d S=\alpha\left(S_{B}, S_{T}\right)
$$

or, see Figure 1(right),

$$
\int_{S_{B}}^{S_{m}} \mathcal{G}\left(S ; S_{B}, S_{T}\right) d S=0 .
$$

Note that if $S_{T} \nearrow S_{T}^{*}\left(S_{B}\right)$, then $S_{m} \nearrow 1$.

The behaviour of the orbits, as demonstrated in [14], is summarized in the following theorem.

Theorem 2.1. Let $0<S_{B}<1$ be fixed and let $S_{B}<S_{T} \leq 1$. Then

(i) If $\tau>\tau_{c, i}$, the orbit leaving $E_{B, i}$ cannot enter $E_{T, i}$ in a monotone way through $\mathcal{H}_{i}$;

(ii) Let $S_{T}<S_{T}^{*}\left(S_{B}\right)$ (or $\left.\alpha\left(S_{B}, S_{T}\right)>0\right)$. Then for any $\tau>0$, the orbit leaving $E_{B, i}$ remains to the left of the line $S=S_{m}$;

(iii) Let $S_{T}>S_{T}^{*}\left(S_{B}\right)$ (or $\left.\alpha\left(S_{B}, S_{T}\right)<0\right)$. Then there exist a $\tau^{*}>0$ such that for $\tau<\tau^{*}$ the orbit leaving $E_{B, i}$ remains to the left of $S=1$. If $\tau>\tau^{*}$, the orbit intersects the line $S=1$ at a negative pressure.

Remark 2.2. (i) The dynamical system (2.6) has three characteristic values for $\tau$ where the behaviour of its solutions changes: $\tau_{c, k}$ for $k=i, d$ defined by (2.16) and $\tau^{*}$ from Theorem 2.1 (iii). By assumption $\tau_{c, i}<\tau_{c, d}$. It is not clear how or if $\tau^{*}$ is ordered with respect to $\tau_{c, i}$ and $\tau_{c, d}$. Therefore we include in the discussion the possibility $\tau^{*}<\tau_{c, i}$ as well as $\tau^{*} \in\left(\tau_{c, i}, \tau_{c, d}\right)$ and $\tau^{*}>\tau_{c, d}$.

(ii) If $\tau<\tau_{c, i}$, the orbit most likely moves in a monotone way through $\mathcal{H}_{i}$ from the saddle $E_{B, i}$ to the sink $E_{T, i}$. However, the nature of the point $E_{T, i}$ for $\tau>\tau_{c, i}$ allows us only to prove statement (i) of Theorem 2.1

(iii) Thus no matter how large $\tau$, the orbit stays to the left of $S=S_{m}$ for $S_{T}<S_{T}^{*}\left(S_{B}\right)$.

(iv) For $S_{T}>S_{T}^{*}\left(S_{B}\right)$ and $\tau$ sufficiently large, the orbit intersects $S=1$ yielding a solution with a plateau at $S=1$ where $u^{\prime}=\mathcal{G}\left(1 ; S_{B}, S_{T}\right)$. This solution is allowed by the extended capillary pressure (1.6). 

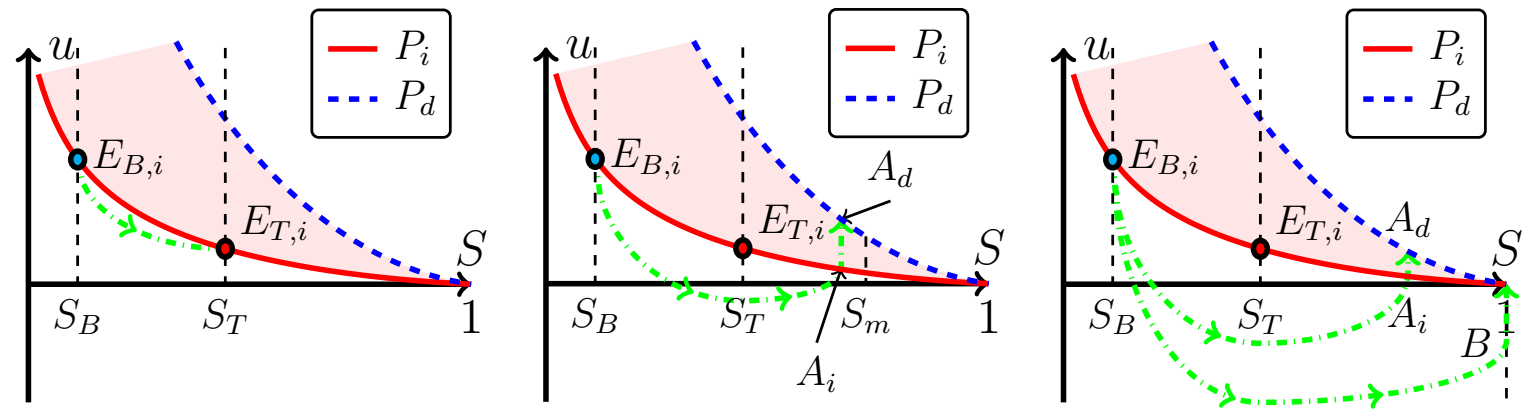

Figure 5: (left) Expected behaviour if $\tau<\tau_{c, i}$; (center) Here $S_{T}<S_{T}^{*}\left(S_{B}\right)$ and orbit remains to the left of $S=S_{m}$. (right) Now $S_{T}>S_{T}^{*}\left(S_{B}\right)$ and $\tau<\tau^{*}$ in the top orbit and $\tau>\tau^{*}$ in the bottom orbit.

The results described by Theorem 2.1 and Remark 2.2 are shown in Figure 5. In case (center) and (right, top) the orbits exit the region $\mathcal{H}_{i}$ at the points $A_{i}=\left(S_{A}, P_{i}\left(S_{A}\right)\right)$, and in case (right, bottom) at the point $B=(S=1, u<0)$.

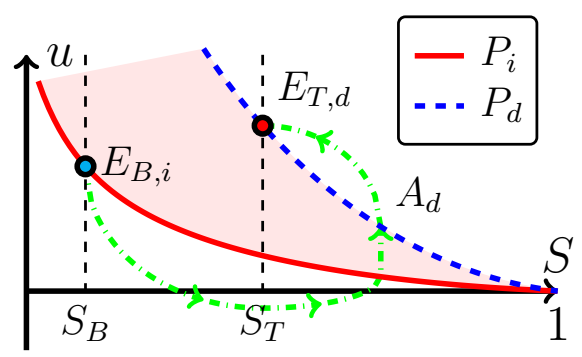

(a)

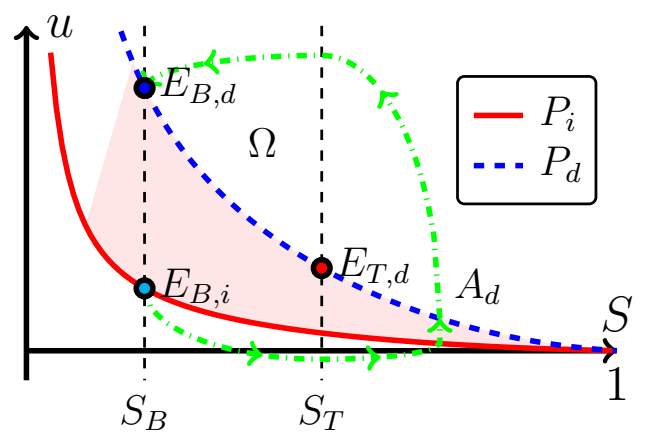

(c)

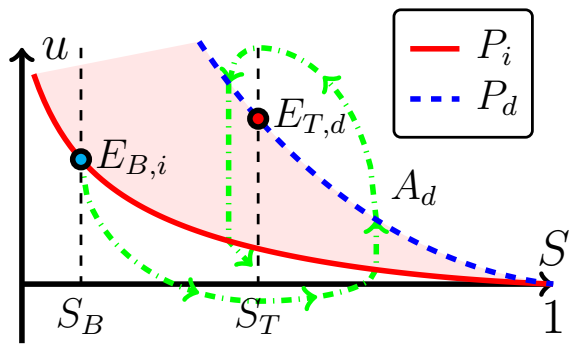

(b)

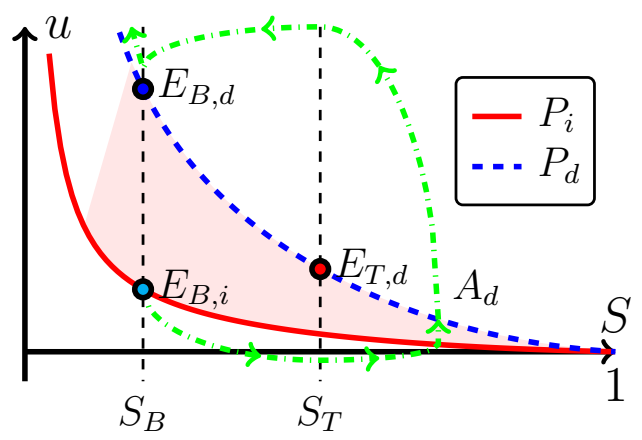

(d)

Figure 6: Four possibilities for orbits starting at the point $A_{d}$ : (a) Orbit moves directly and monotonically to $E_{T, d}$ as $\zeta \rightarrow \infty$; (b) orbit passes $S=S_{T}$ and returns to the hysteretic region $\mathcal{H}$; (c) orbit passes $S=S_{T}$ and ends up at $E_{B, d}$ as $\zeta \rightarrow \infty$; (d) Orbit passes first $S=S_{T}$ and then $S=S_{B}$ above $E_{B, d}$.

Since $\mathcal{F}=0$ in $\mathcal{H}$, the orbits at $A_{i}$ move vertically upwards until they reach the 
drainage curve $P_{d}$ at $A_{d}=\left(S_{A}, P_{d}\left(S_{A}\right)\right)$. Using again results from [14], we are left with four possibilities that are shown in Figure 6.

Cases (c) and (d) are ruled out by the following divergence argument (see also $[4,14]$ ). Write system (2.6) as

$$
\vec{u}^{\prime}=\vec{R}(\vec{u})
$$

where

$$
\vec{u}=\left(\begin{array}{c}
S \\
u
\end{array}\right) \text { and } \vec{R}(\vec{u})=\left(\begin{array}{c}
\frac{1}{c \tau} \mathcal{F}(S, u) \\
\mathcal{G}\left(S ; S_{B}, S_{T}\right)
\end{array}\right)
$$

and observe that

$$
\operatorname{div} \vec{R}=\frac{1}{c \tau} \frac{\partial \mathcal{F}}{\partial S} \leq 0
$$

as

$$
\frac{\partial \mathcal{F}}{\partial S}= \begin{cases}P_{i}^{\prime}(S) & \text { when } u \in \mathcal{H}_{i}, \\ 0 & \text { when } u \in \mathcal{H}, \\ P_{d}^{\prime}(S) & \text { when } u \in \mathcal{H}_{d}\end{cases}
$$

Let $\Omega$ in Figure 6(c) denote the region enclosed by the orbit running from $E_{B, i}$ to $E_{B, d}$ and the vertical segment at $S=S_{B}$. Then the divergence theorem applied to $\vec{R}$ in $\Omega$ gives a contradiction. The same argument applies to case (d) (see [14]). Hence we are left with cases (a) and (b) in which the orbit is captured in a region around the segment $\overline{E_{T, i} E_{T, d}}$. In case (a) the solution moves monotonically from $A_{d}$ to the equilibrium point $E_{T, d}$. Clearly this is only possible when $\tau<\tau_{c, d}$ (and $E_{T, d}$ is a sink). In case (b) the orbit goes at least once around $\overline{E_{T, i} E_{T, d}}$ and it may reach $E_{T, i}\left(\right.$ if $\left.\tau<\tau_{c, i}\right)$ or $E_{T, d}\left(\right.$ if $\tau<\tau_{c, d}$ ) after finitely many rotations. If $\tau>\tau_{c, d}>\tau_{c, i}$, the nature of the points $E_{T, k},(k=i, d)$ prevents this behaviour.

When the orbit exits at point $B$ as in Figure 5(right), it moves vertically upwards with $S=1$ and $u^{\prime}=\mathcal{G}\left(1 ; S_{B}, S_{T}\right)>0$ until it reaches $S=1, u=0$. Then we repeat the previous argument.

With these observations in mind, we now investigate the behaviour of the orbits near $S=S_{T}$. We first consider

$\tau>\tau_{c, d}\left(>\tau_{c, i}\right)$

Then the endpoints $E_{T, i}$ and $E_{T, d}$ are stable spirals, implying that the behaviour as sketched in Figure 6(a) cannot occur. Hence we are left with Figure 6(b) as the only option. Since an orbit cannot intersect itself, it will be captured in a neighborhood of the segment $\overline{E_{T, i} E_{T, d}}$. Moreover, since orbits move vertically through $\mathcal{H}$, they cannot reach an interior point of $\overline{E_{T, i} E_{T, d}}$ and the corresponding saturation has plateaus below and 
above $S_{T}$. Clearly there are infinitely many of these plateaus, otherwise the orbits would end up in $E_{T, i}$ or $E_{T, d}$ after finitely many rotations.

We are now in a position to prove

Lemma 2.1. Let $0<S_{B}<S_{T}<1$ and $\tau>\tau_{c, d}$. Further, for a given orbit satisfying (2.6), let $\left\{S_{n}^{ \pm}\right\}_{n=1}^{\infty}$ denote the saturation plateaus above (+) and below (-) $S_{T}$. Then

(i) $S_{n}^{-}<S_{n+1}^{-}<S_{T}$ for $n \geq 1$ and $\lim _{n \rightarrow \infty} S_{n}^{-} \nearrow S_{T}$;

(ii) $S_{n}^{+}>S_{n+1}^{+}>S_{T}$ for $n \geq 1$ and $\lim _{n \rightarrow \infty} S_{n}^{+} \searrow S_{T}$.

Proof. We only show (i). The monotonicity is a consequence of the fact that orbits cannot intersect themselves. Now suppose that a $\delta>0$ exists such that

$$
S_{n}^{-} \leq S_{T}-\delta \text { for all } n \geq 1
$$

Let,

$$
\mathcal{R}_{\delta}=\left\{(S, u): S_{T}-\delta<S<S_{T}, P_{i}(S)<u<P_{d}\left(S_{T}-\delta\right)\right\},
$$

see Figure 7. Since the orbit is confined to a neighborhood of $\overline{E_{T, i} E_{T, d}}$ and rotates around $\mathcal{R}_{\delta}$, it must converge, as $\zeta \rightarrow \infty$, to a limit cycle $C$. Let $\Omega$ denote the region enclosed by $C$. Clearly $\Omega \supseteq \mathcal{R}_{\delta}$. Since $\operatorname{div} \vec{R} \leq 0$ in $\Omega$, in particular $\operatorname{div} \vec{R}=\frac{1}{c \tau} P_{d}^{\prime}(S)<0$ in $\left\{(S, u): S_{T}-\delta<S<S_{T}, P_{d}(S)<u<P_{d}\left(S_{T}-\delta\right)\right\}$, the divergence theorem applied to $\bar{R}$ in $\Omega$ yields a contradiction. Hence $\delta=0$.

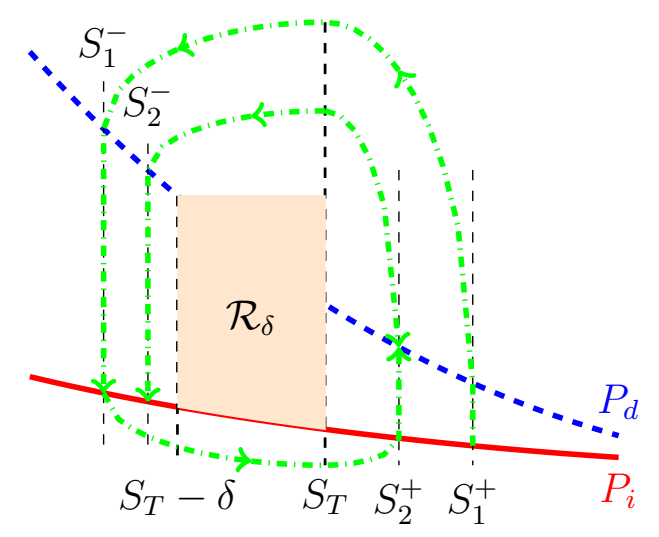

Figure 7: Rotation of orbit around $\mathcal{R}_{\delta}$.

When the orbit crosses the hysteresis domain $\mathcal{H}$ at some $S=\hat{S} \in(0,1)$, the pressure equation satisfies

$$
u^{\prime}=\mathcal{G}\left(\hat{S} ; S_{B}, S_{T}\right)
$$


Hence, the $\zeta$-interval $(\ell)$ in which this takes place has length

$$
\mu(\ell)=\frac{P_{d}(\hat{S})-P_{i}(\hat{S})}{\mathcal{G}\left(\hat{S} ; S_{B}, S_{T}\right)} .
$$

Thus as a consequence of Lemma 2.1, we have

Corollary 2.1. Corresponding to the saturation plateaus $\left\{S_{n}^{ \pm}\right\}_{n=1}^{\infty}$, there exist $\zeta$-intervals $\left\{\ell_{n}^{ \pm}\right\}_{n=1}^{\infty}$ satisfying

(i) $\ell_{n}^{-}$is between $\ell_{n}^{+}$and $\ell_{n+1}^{+}$.

(ii) $\mu\left(\ell_{n}^{ \pm}\right)=\frac{P_{d}\left(S_{n}^{ \pm}\right)-P_{i}\left(S_{n}^{ \pm}\right)}{\mathcal{G}\left(S_{n}^{ \pm} ; S_{B}, S_{T}\right)}$.

(iii) $\lim _{n \rightarrow \infty} \mu\left(\ell_{n}^{ \pm}\right)=\infty$.

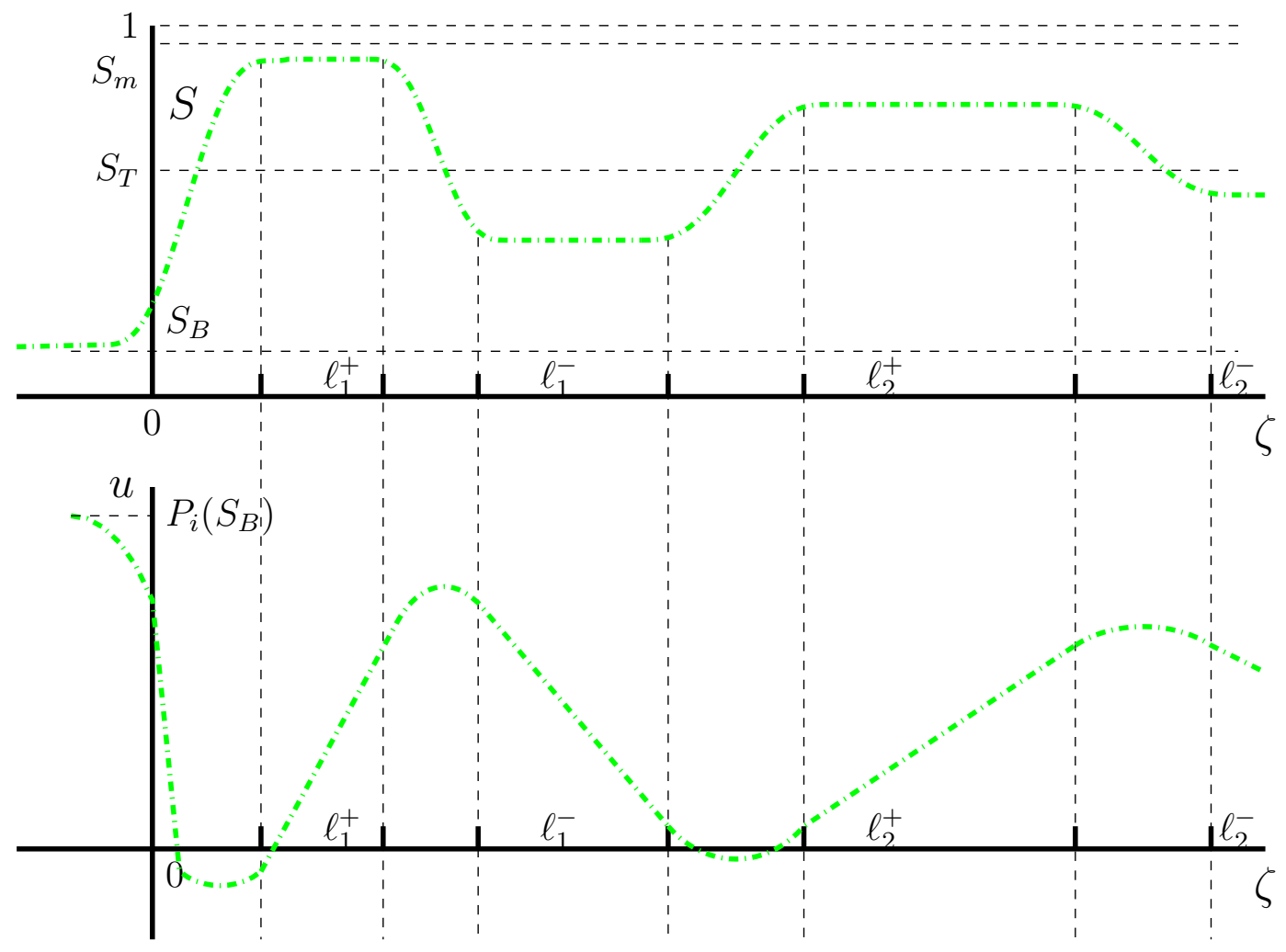

Figure 8: Sketch of saturation and pressure behaviour. Here $S_{T}<S_{T}^{*}\left(S_{B}\right)$ implying $S<S_{m}<1$. If $S_{T}>S_{T}^{*}\left(S_{B}\right)$ and $\tau>\tau^{*}$, the first saturation plateau occurs at $S=1$. 
Proof. (i) This is immediate from the orbit rotating around $\overline{E_{T, i} E_{T, d}}$.

(ii) The expression follows directly from (2.21).

(iii) This is a consequence of Lemma 2.1, (ii), $P_{d}(S)>P_{i}(S)$ for $0<S<1$ and $\mathcal{G}\left(S_{T} ; S_{B}, S_{T}\right)=0$.

The behaviour described by Lemma 2.1 and Corollary 2.1 is sketched in Figure 8.

Remark 2.3. Lemma 2.1 implies that the pressure $u(\zeta)$ oscillates between $P_{i}\left(S_{T}\right)$ and $P_{d}\left(S_{T}\right)$ as $\zeta \rightarrow \infty$. But Corollary 2.1 shows that $\lim _{\zeta \rightarrow \infty} u^{\prime}(\zeta)=0$.

$\underline{\tau} \leq \tau_{c, d}$

Then either $E_{T, d}$ (if $\tau_{c, i}<\tau \leq \tau_{c, d}$ ) or both $E_{T, i}$ and $E_{T, d}\left(\right.$ if $\tau \leq \tau_{c, i}$ ) are a sink. This gives the following result.

Lemma 2.2. Let $0<S_{B}<S_{T}<1$ and $\tau \leq \tau_{c, d}$. Then for any orbit satisfying (2.6), we have:

(i) If $0<\tau \leq \tau_{c, i}$, it moves directly and monotonically through $\mathcal{H}_{i}$ from $E_{B, i}$ to $E_{T, i}$ or it rotates finitely many times around $\overline{E_{T, i} E_{T, d}}$ and ends up in $E_{T, i}$ or $E_{T, d}$;

(ii) If $\tau_{c, i}<\tau \leq \tau_{c, d}$, it ends up in $E_{T, d}$ after a finite number of passages through $\mathcal{H}$.

Proof. (i) If the orbit does not move directly from $E_{B, i}$ to $E_{T, d}$ through $\mathcal{H}_{i}$, it has to cross the hysteretic domain $\mathcal{H}$ or it has a saturation plateau at $S=1$. Then we argue as in Lemma 2.1 to show that the orbit must approach $\overline{E_{T, i} E_{T, d}}$ as $\zeta \rightarrow \infty$. The fact that both $E_{T, i}$ to $E_{T, d}$ are sinks implies that the orbit will be captured by one of these points after finitely many rotations.

(ii) Now only $E_{T, d}$ is a sink. Arguing as in (i) gives the result.

In the discussion and figures so far $S_{T}<1$ has been considered. If $S_{T}=1$, the orbit cannot intersect the imbibition pressure curve at $S_{T}<1$. This follows from the flow direction as shown in Figure 9. Furthermore, each point of the half line $\{S=1, u \leq 0\}$ is an equilibrium point of (2.6) with the extended capillary pressure (1.6). In this case the existence result is given by 


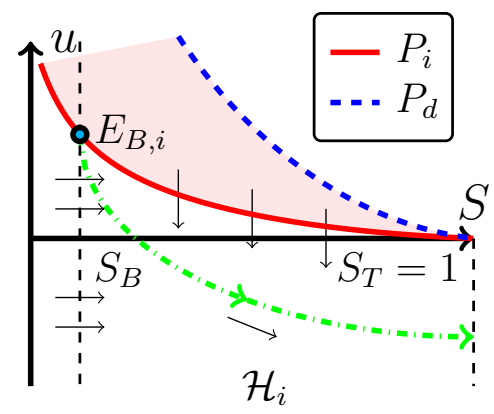

Figure 9: Sketch of flow direction when $S_{T}=1$.

Lemma 2.3. Let $0<S_{B}<S_{T}=1$.

(i) If $P_{i}^{\prime}(1)=0$, then for any $\tau>0$ the solution of (2.6) is in $\mathcal{H}_{i}$ and runs from $E_{B, i}$ as $\zeta \rightarrow-\infty$ to a point $\left(S=1, u=u_{\tau}<0\right)$ at a finite value of $\zeta=\zeta_{\tau}$. For $\zeta \geq \zeta_{\tau}$ the solution is given by $S(\zeta)=1$ and $u(\zeta)=u_{\tau}$. The pressure value $u_{\tau}$ decreases in $\tau$ and has the lower bound $u_{\tau} \geq-\sqrt{2 c K \tau}$, where $K=-\alpha\left(S_{B}, 1\right)>0$. Furthermore, $\lim _{\zeta \nearrow \zeta_{\tau}} S^{\prime}(\zeta)=-\frac{u_{\tau}}{c \tau}$ and $\lim _{\zeta \nearrow \zeta_{\tau}} u^{\prime}(\zeta)=0 ;$

(ii) if $P_{i}^{\prime}(1)<0$, then the solution behaves as in (i) when $\tau>\tau_{c, i}$. For $\tau \leq \tau_{c, i}$ it may end up in $(1,0)$ as $\zeta \rightarrow \infty$.

Proof. Since the orbit belongs to $\mathcal{H}_{i}$, equations (2.6) reduce to

$$
\begin{aligned}
S^{\prime} & =\frac{1}{c \tau}\left(P_{i}(S)-u\right), \\
u^{\prime} & =\mathcal{G}\left(S ; S_{B}, 1\right) .
\end{aligned}
$$

(i) With $P_{i}^{\prime}(1)=0$, the eigenvalues at the point $(1,0)$ are given by

$$
\lambda_{k}= \pm j \sqrt{c \tau \mathcal{G}^{\prime}\left(1 ; S_{B}, 1\right)},
$$

where $j=\sqrt{-1}$. This means that for any $\tau>0$, the orbits cannot reach $(1,0)$ as $\zeta \rightarrow \infty$. Hence they must exit $\mathcal{H}_{i}$ at some point $\left(S=1, u=u_{\tau}<0\right)$ for some finite $\zeta=\zeta_{\tau}$. For $\zeta \geq \zeta_{\tau}$ we extend the solution by the equilibrium $\left(S=1, u=u_{\tau}\right)$. The properties of the pressure value $u_{\tau}$ are demonstrated along the lines of the proof of [14, Theorem 3.1(b) and Proposition 4.2]. Finally, letting $\zeta \nearrow \zeta_{\tau}$ in equations (2.22) gives

$$
\lim _{\zeta \nearrow \zeta_{\tau}} S^{\prime}(\zeta)=\frac{1}{c \tau} \lim _{\zeta \zeta_{\tau}}\left(P_{i}(S(\zeta))-u(\zeta)\right)=-\frac{u_{\tau}}{c \tau}
$$

and

$$
\lim _{\zeta \nearrow \zeta_{\tau}} u^{\prime}(\zeta)=\lim _{\zeta \nearrow \zeta_{\tau}} \mathcal{G}\left(S(\zeta) ; S_{B}, 1\right)=0 .
$$

(ii) If $P_{i}^{\prime}(1)<0$, the orbit behaves as in (i) when $\tau>\tau_{c, i}$. For $\tau \leq \tau_{c, i}$ the point $(1,0)$ is a sink and thus the orbit may end up at that point as $\zeta \rightarrow \infty$. In particular this happens for very small $\tau$ values. 


\section{2 $f \in L^{1}(0,1)$}

When the capillary functions in (1.4) is non-constant, one uses (2.12) to recast (2.6) in the equivalent form (2.14), where $f$ is now removed from the equations. If $f \in L^{1}(0,1)$, then $0<Y<Y(1)=\int_{0}^{1} f(\varrho) d \varrho$. The behaviour in terms of $(Y, u)$ in the strip $\{(Y, u)$ : $0<Y \leq Y(1), u \in \mathbb{R}\}$ is similar to the behaviour of $(S, u)$ with $f(S)=1$, as discussed in Section 2.1.

In terms of the transformed nonlinearities $\tilde{\mathcal{F}}, \tilde{G}$ and $\tilde{P}_{k}(k=i, d)$, see definitions below (2.14), the characteristic $\tau$-values in terms of $Y$ are

$$
\tilde{\tau}_{c, k}=\frac{\left(\tilde{P}_{k}^{\prime}\left(Y_{T}\right)\right)^{2}}{4 c \tilde{\mathcal{G}}^{\prime}\left(Y_{T} ; Y_{B}, Y_{T}\right)} \quad(k=i, d) .
$$

Likewise,

$$
\tilde{\alpha}\left(Y_{B}, Y_{T}\right)=\int_{Y_{B}}^{Y(1)} \tilde{\mathcal{G}}\left(\varrho ; Y_{B}, Y_{T}\right) d \varrho
$$

and

$$
\int_{Y_{B}}^{Y_{m}} \tilde{\mathcal{G}}\left(\varrho ; Y_{B}, Y_{T}\right) d \varrho=0 .
$$

Transforming these expressions back to the original $(S, u)$ variables now gives

$$
\begin{aligned}
& \tau_{c, k}=\frac{\left(P_{k}^{\prime}\left(S_{T}\right)\right)^{2}}{4 c f\left(S_{T}\right) \mathcal{G}^{\prime}\left(S_{T} ; S_{B}, S_{T}\right)} \quad(k=i, d), \\
& \alpha\left(S_{B}, S_{T}\right)=\int_{S_{B}}^{1} \mathcal{G}\left(\varrho ; S_{B}, S_{T}\right) f(\varrho) d \varrho, \\
& \int_{S_{B}}^{S_{m}} \mathcal{G}\left(\varrho ; S_{B}, S_{T}\right) f(\varrho) d \varrho=0 .
\end{aligned}
$$

Given these new definitions one can repeat the arguments of Section 2.1 to obtain similar results. Clearly $S_{T}^{*}\left(S_{B}\right)$, see Figure 4 , depends on $f$ through the expression (2.27). Also the value $\tau^{*}$ from Theorem 2.1 will change with $f$.

Remark 2.4. Let $f_{1}, f_{2} \in L^{1}(0,1)$ and $f_{1}<f_{2}$ in $(0,1)$. Then the critical $\tau$-values $(2.26)$ are ordered as well:

$$
\tau_{c, k, 1}>\tau_{c, k, 2} \quad(k=i, d)
$$

Hence in case 2, overshoot is expected to occur for smaller values of $\tau$.

\section{$2.3 f \notin L^{1}\left(S_{B}, 1\right)$}

Again we use transformation (2.12) and the corresponding redefinitions of the nonlinearities. Borrowing ideas from [14, Section 4.2], we distinguish the cases $S_{T}<1$ and $S_{T}>1$. 
$\underline{S_{T}<1}$. Then there always exists $S_{m} \in\left(S_{T}, 1\right)$ satisfying (2.28). To see this, observe that for any $\delta \in\left(0, \frac{1-S_{T}}{2}\right)$

$$
\begin{aligned}
& \int_{S_{B}}^{1-\delta} f \mathcal{G} d S=\int_{S_{B}}^{\left(S_{T}+1\right) / 2} f \mathcal{G} d S+\int_{\left(S_{T}+1\right) / 2}^{1-\delta} f \mathcal{G} d S \\
& \geq \int_{S_{B}}^{\left(S_{T}+1\right) / 2} f \mathcal{G} d S+\mathcal{G}\left(\left(S_{T}+1\right) / 2 ; S_{B}, S_{T}\right) \int_{\left(S_{T}+1\right) / 2}^{1-\delta} f d S .
\end{aligned}
$$

Since $\lim _{\delta \rightarrow 0} \int_{S_{B}}^{1-\delta} f \mathcal{G} d S=\infty$ and as $\int_{S_{B}}^{S_{T}} f \mathcal{G} d S<0$ the assertion follows. As $S_{m}<1$, Theorem 2.1 further implies that $S(\zeta) \leq S_{m}<1$ for all $\tau>0$ and $\zeta \in \mathbb{R}$. Hence, $f(S(\zeta))$ is bounded away from $\infty$ for $\zeta \in \mathbb{R}$. Consequently, the results presented in Lemma 2.1-2.2 and Corollary 2.1 remain valid and depending upon the value of $\tau$, the orbits can either end up in $E_{T, i}$ or $E_{T, d}$ or rotate infinitly many times around the segment $\overline{E_{T, i} E_{T, d}}$ while approaching it.

$\underline{S_{T}=1}$. Recall that each point on the half-line $\{S=1, u \leq 0\}$ is an equilibrium point. In fact the extension of $P_{k}(k=i, d)$ as in (1.8), is not required in this case, as $\left(P_{k}(S)-u\right) / c \tau f \rightarrow 0$ for $S \nearrow 1, k=i, d$. This implies that an orbit $(S, u)$ leaving $E_{B, i}$ cannot cross the line $\{S=1, u \leq 0\}$. Moreover, $u^{\prime}(\zeta)=\mathcal{G}\left(S(\zeta) ; S_{B}, 1\right)<0$ and $S^{\prime}(\zeta)>0$ for all $\zeta \in \mathbb{R}$. This along with the direction of orbits shown in Figure 9 implies that there are only three options for the orbit $(S, u)$ as $\zeta \rightarrow \infty$ : (i) $(S, u) \rightarrow(1,0)$, (ii) $(S, u) \rightarrow\left(1, u_{\tau}\right)$ with $-\infty<u_{\tau}<0$ or $($ iii $)(S, u) \rightarrow(1,-\infty)$.

From [14, Theorem 4.4] it follows that (iii) occurs if and only if

$$
\int_{S_{B}}^{1} \mathcal{G}\left(\varrho ; S_{B}, 1\right) f(\varrho) d \varrho=-\infty .
$$

However, since $\mathcal{G}\left(1 ; S_{B}, 1\right)=0$ this integral may be finite. As $\mathcal{G}\left(S ; S_{B}, 1\right)$ is Lipschitz and negative for $S>S_{B}$, the integral is finite if

$$
\int_{0}^{1}(1-\varrho) f(\varrho) d \varrho<\infty .
$$

In this case, one defines $\alpha\left(S_{B}, 1\right)$ by putting $S_{T}=1$ in $(2.27)$. As $\alpha\left(S_{B}, 1\right)>-\infty$, $(S, u) \rightarrow\left(1, u_{\tau}\right)$ as $\zeta \rightarrow \infty$ for some $-\infty<u_{\tau} \leq 0$. From [14, Proposition 4.2b], one further obtains that there exists $\hat{\tau} \geq 0$, such that if $\tau>\hat{\tau}$ then $u_{\tau}<0$. Hence, (i) is only possible for small values of $\tau$. Moreover, in (ii), $u_{\tau}$ is bounded below by the estimate given in Lemma 2.3.

We summarize these findings in the following lemma

Lemma 2.4. Let $f \notin L^{1}(0,1)$ and assume that $\tau>0, S_{B}>0$ and $S_{T} \in\left(S_{B}, 1\right]$. Further, if $(1-\varrho) f(\varrho) \in L^{1}(0,1)$, let $\alpha\left(S_{B}, 1\right):=\int_{S_{B}}^{1} \mathcal{G}\left(\varrho ; S_{B}, 1\right) f(\varrho) d \varrho$. Then for orbit $(S(\zeta), u(\zeta))$ satisfying (2.6) we have: 
(i) If $S_{T}<1$, results presented in Lemmas 2.1-2.2 and Corollary 2.1 remain valid and $S(\zeta) \leq S_{m}<1$ for all $\zeta \in \mathbb{R}$, where $S_{m}$ is defined by (2.28).

(ii) If $S_{T}=1$, there can be two cases:

(ii.1) If $(1-\varrho) f(\varrho) \notin L^{1}(0,1)$, then $S(\zeta) \nearrow 1$ and $u(\zeta) \rightarrow-\infty$ as $\zeta \rightarrow \infty$.

(ii.2) If $(1-\varrho) f(\varrho) \in L^{1}(0,1)$, then a $\hat{\tau} \geq 0$ exists such that if $\tau \leq \hat{\tau}$ then $(S, u) \rightarrow$ $(1,0)$ as $\zeta \rightarrow \infty$; if $\tau>\hat{\tau}$ then $(S, u) \rightarrow\left(1, u_{\tau}\right)$ as $\zeta \rightarrow \infty$ for some $u_{\tau} \in$ $(-\infty, 0]$. Here $u_{\tau}$ satisfies $u_{\tau} \geq-\sqrt{-2 c \alpha\left(S_{B}, 1\right) \tau}>-\infty$.

Figure 10 shows a schematic diagram outlining the cases.

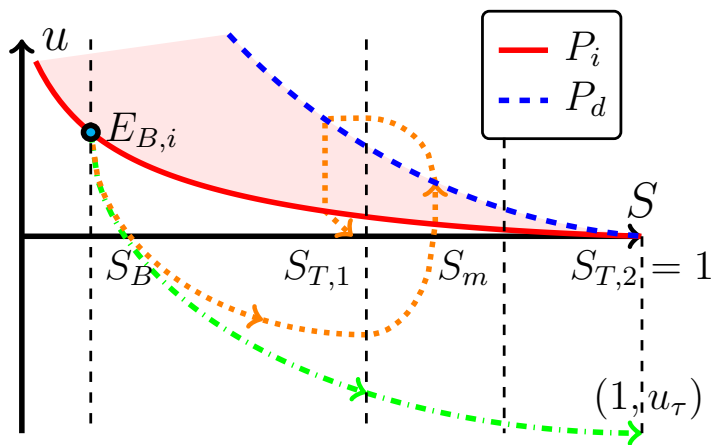

Figure 10: Behaviour of orbits for $f \notin L^{1}(0,1)$. The dotted (orange) line represents the orbit for $S_{T}=S_{T, 1}<1$. The dashdotted line (green) represents the orbit for $S_{T}=S_{T, 2}=$ 1 , when $(1-\varrho) f(\varrho) \in L^{1}(0,1)$ and $\tau>\hat{\tau}$.

\section{$3 \quad$ Travelling waves with combined pressure (1.6)}

In the previous section we discussed travelling waves arising from the combined pressure model with play-type hysteresis (1.4). The scanning curves for the playtype hysteresis model are vertical, whereas scanning curves obtained from experiments are inclined $[1,8]$. As pointed out in [6], this may lead to inaccurate predictions. The combined model with extended play-type hysteresis given by (1.6), incorporates non-vertical scanning curves that can be fitted to match experimental observations, see [6]. Hence, it is interesting to study how the behaviour of the infiltration fronts changes if one uses the extended model to describe hysteresis.

As seen from Section 2, for sufficiently large values of $\tau$, the orbit $(S(\zeta), u(\zeta))$ revolves around the line segment $\overline{E_{T, i} E_{T, d}}$ infinitely many times. From a physical point of view, this means that for an infinitely long column, one observes infinitely many decaying oscillations trailing behind the first overshoot. This behaviour is indicated in Figure 8. However, few to no oscillations are observed behind the first overshoot in experimentally 
obtained breakthrough curves $[2,5]$ even for long columns and large injection rates. In this section we explain this by considering non-vertical scanning curves.

In the subsequent discussions, we assume that the inclination of the scanning curves satisfies

$$
\min \left\{P_{i}^{\prime}(S), P_{d}^{\prime}(S)\right\}>-\frac{1}{\varepsilon}, \text { for all } S_{B} \leq S \leq 1
$$

As shown in [6], consistency of the extended model requires that (3.1) should be satisfied for all $S \in(0,1]$. Hence, for more general problems a non-constant $\varepsilon$ needs to be considered. However, since travelling waves satisfy $S \geq S_{B}$, and since $P_{i}^{\prime}, P_{d}^{\prime}$ are bounded in $S \in\left[S_{B}, 1\right]$ it suffices to consider (3.1) for $\varepsilon$ sufficiently small.

Substituting the travelling wave form (2.1) into (1.1) and (1.7), and applying conditions (2.2) and (2.4) we obatin the same Rankine-Hugoniot wave speed $c$ and the same function $\mathcal{G}\left(S ; S_{B}, S_{T}\right)$, see $(2.5)$ and $(2.8)$. The extended system now reads:

$$
\begin{aligned}
S^{\prime} & =\frac{1}{c \tau f(S)} E(S, u), \\
u^{\prime} & =\mathcal{G}\left(S ; S_{B}, S_{T}\right),
\end{aligned}
$$

where

$$
E(S, u)=\left\{\begin{array}{lc}
P_{d}(S)-u & \text { when } u>P_{d}(S)+\varepsilon \tau c f(S) \mathcal{G}\left(S ; S_{B}, S_{T}\right), \\
-c \varepsilon \tau f(S) \mathcal{G}\left(S ; S_{B}, S_{T}\right) & \text { when } P_{i}(S)+\varepsilon \tau c f(S) \mathcal{G}\left(S ; S_{B}, S_{T}\right) \\
& \leq u \leq P_{d}(S)+\varepsilon \tau c f(S) \mathcal{G}\left(S ; S_{B}, S_{T}\right), \\
P_{i}(S)-u & \text { when } u<P_{i}(S)+\varepsilon \tau c f(S) \mathcal{G}\left(S ; S_{B}, S_{T}\right) .
\end{array}\right.
$$

Equations (3.2), with expression (3.3), shows that $f(S)$ cannot be eliminated by transformation (2.12). Therefore, for simplicity we restrict ourselves here to the case $f(S)=1$ only. Then the system becomes

$$
\begin{aligned}
S^{\prime} & =\frac{1}{c \tau} \mathcal{E}(S, u), \\
u^{\prime} & =\mathcal{G}\left(S ; S_{B}, S_{T}\right),
\end{aligned}
$$

Here

$$
\mathcal{E}(S, u)= \begin{cases}P_{d}(S)-u & \text { when } u>\hat{u}(S) \\ -c \varepsilon \tau \mathcal{G}\left(S ; S_{B}, S_{T}\right) & \text { when } \check{u}(S) \leq u \leq \hat{u}(S) \\ P_{i}(S)-u & \text { when } u<\check{u}(S) .\end{cases}
$$

where $\check{u}, \hat{u}:(0,1] \rightarrow \mathbb{R}$ are given by

$$
\check{u}(S)=P_{i}(S)+\varepsilon \tau c \mathcal{G}\left(S ; S_{B}, S_{T}\right) \text { and } \hat{u}(S)=P_{d}(S)+\varepsilon \tau c \mathcal{G}\left(S ; S_{B}, S_{T}\right) .
$$


Note that for each $\delta>0, \mathcal{E}(S, u)$ is uniformly Lipschitz continuous in the strip $\{\delta \leq S \leq$ $1\}$. Similar to the sets $\mathcal{H}, \mathcal{H}_{i}, \mathcal{H}_{d}$ in (2.9), we now divide the strip $\{0<S<1\}$ into

$$
\begin{aligned}
\hat{\mathcal{H}} & =\{u>\hat{u}(S)\}, \\
\overline{\mathcal{H}} & =\{\check{u}(S)<u<\hat{u}(S)\}, \\
\check{\mathcal{H}} & =\{u<\check{u}(S)\} .
\end{aligned}
$$

For given $0<S_{B}<S_{T} \leq 1$, the parameters of the system are $\varepsilon$ and $\tau$. In the region $\overline{\mathcal{H}}$ one has

$$
S^{\prime}=-\varepsilon \mathcal{G}\left(S ; S_{B}, S_{T}\right)=-\varepsilon u^{\prime},
$$

implying indeed scanning curves with inclination $-1 / \varepsilon$ in $\overline{\mathcal{H}}$.

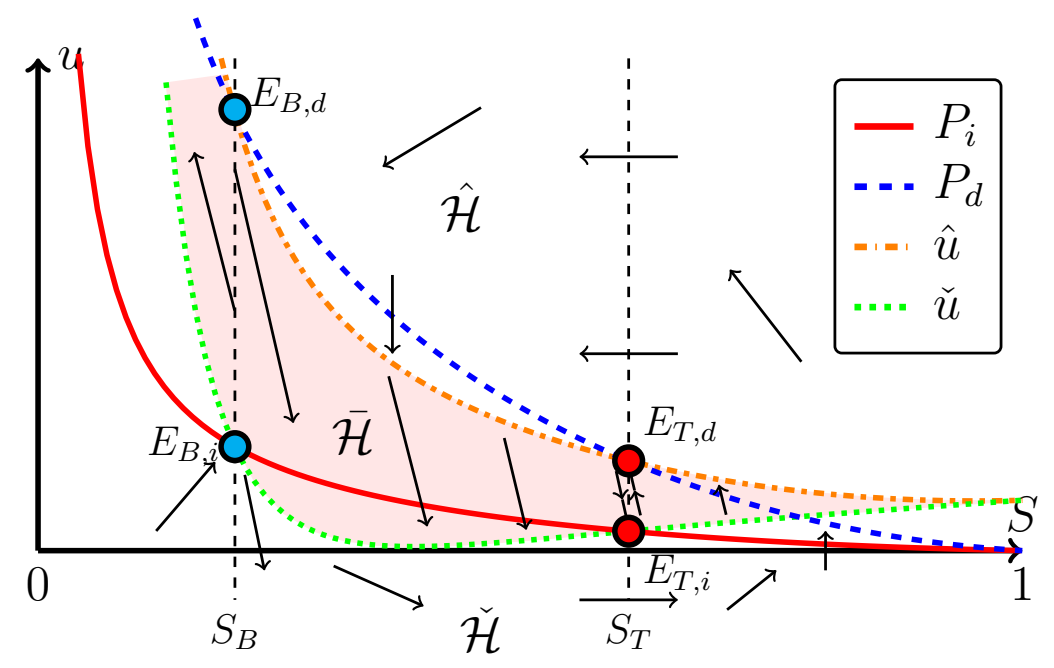

Figure 11: The sets (3.6) and the curves (3.5) in the strip $\{0<S \leq 1\}$. The arrows indicate the direction for increasing $\zeta$ of solutions of (3.4).

As in play-type hysteresis, each point of the segments $\overline{E_{B, i} E_{B, d}}$ and $\overline{E_{T, i} E_{T, d}}$ is an equilibrium point. From equation (3.7) and the sign of $\mathcal{G}\left(S ; S_{B}, S_{T}\right)$ it follows that interior points of $\overline{E_{B, i} E_{B, d}}$ are unstable, whereas interior points of $\overline{E_{T, i} E_{T, d}}$ are stable. Further, the nature of the end points $\left\{E_{B, k}, E_{T, k}\right\},(k=i, d)$ is as in Section 2: $E_{B, k}$ are saddles and $E_{T, k}$ are stable nodes and/or stable spirals depending on the value of $\tau$. This classifies all equilibrium points of (3.4). 


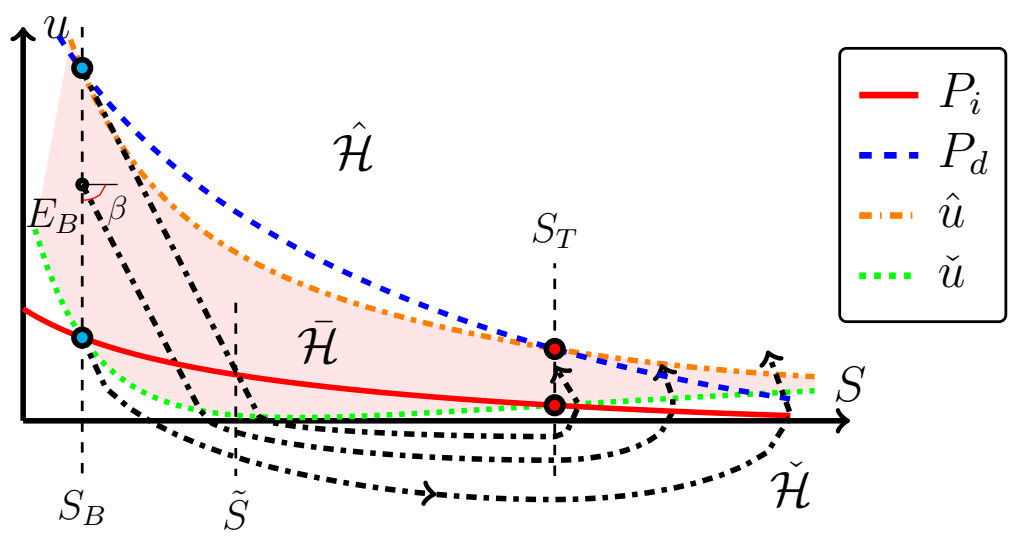

Figure 12: Possible orbits starting, as $\zeta \rightarrow-\infty$, from points $E_{B} \in \overline{E_{B, i} E_{B, d}}$. Here $E_{B}=\left(S_{B}, u_{B}\right)$ and $\tan \beta=-\frac{1}{\varepsilon}$.

The main result of this section is to show existence of solutions of (3.4), subject to conditions (2.2) and (2.4), in the form of orbits that connect arbitrarily chosen points $E_{B} \in \overline{E_{B, i} E_{B, d}}($ as $\zeta \rightarrow-\infty)$ to corresponding points $E_{T} \in \overline{E_{T, i} E_{T, d}}$ (as $\zeta \rightarrow+\infty$ ). We shall give the proof in context of Figure 11. This figure shows the regions $\hat{\mathcal{H}}, \overline{\mathcal{H}}, \check{\mathcal{H}}$, the functions $\check{u}, \hat{u}$ and the general flow directions of solution orbits $(S, u)$. To support the proof we added Figure 12 which enlarges the situation between the segments $\overline{E_{B, i} E_{B, d}}$ and $\overline{E_{T, i} E_{T, d}}$.

Let $u_{B} \in\left[P_{i}\left(S_{B}\right), P_{d}\left(S_{B}\right)\right]$ and let

$$
\ell\left(S ; u_{B}\right)=u_{B}-\frac{1}{\varepsilon}\left(S-S_{B}\right) \text { for } S>S_{B}
$$

With reference to Figure 11 and Figure 12 we assume that $\varepsilon$ and $\tau$ are such that

$$
\ell\left(S ; P_{d}\left(S_{B}\right)\right) \leq \hat{u}(S) \text { for } S_{B} \leq S \leq 1
$$

and

$$
\check{u}^{\prime}(S) \geq-\frac{1}{\varepsilon} .
$$

As $\ell\left(S_{B}, P_{d}\left(S_{B}\right)\right)=P_{d}\left(S_{B}\right)$, a sufficient condition for (3.9) to be true is

$$
1+\varepsilon \hat{u}^{\prime}(S)=1+\varepsilon P_{d}^{\prime}(S)+c \tau \varepsilon^{2} \mathcal{G}^{\prime}\left(S ; S_{B}, S_{T}\right) \geq 0,
$$

for $S_{B} \leq S \leq 1$. Similarly for (3.10) the condition is

$$
1+\varepsilon \check{u}^{\prime}(S)=1+\varepsilon P_{i}^{\prime}(S)+c \tau \varepsilon^{2} \mathcal{G}^{\prime}\left(S ; S_{B}, S_{T}\right) \geq 0
$$

for $S_{B} \leq S \leq 1$. From (3.1), as $1+\varepsilon P_{k}{ }^{\prime}(S)>0(k=i, d)$, Conditions (3.11) are satisfied if $\varepsilon^{2} \tau$ is small enough. Moreover, because $\mathcal{G}^{\prime}>0$ for $S \geq S_{l}$ (see Figure 1) it follows from 


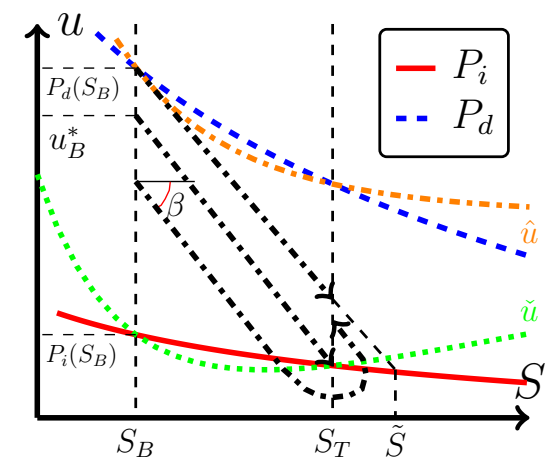

Figure 13: Sketch of orbits when $S_{T}<\tilde{S}$. Here, $\tan (\beta)=-\frac{1}{\varepsilon}$.

(3.1) that Conditions (3.11) are automatically satisfied for $S>S_{l}$. Hence, Conditions (3.11) are only to be assumed for $S \in\left[S_{B}, S_{l}\right]$.

Observe that from (3.1),

$$
\ell\left(1 ; P_{d}\left(S_{B}\right)\right)<0=\lim _{S \nearrow 1} P_{i}(S)
$$

Using (3.1) and (3.12) it follows that there exists a unique saturation value $\tilde{S} \in\left(S_{B}, 1\right)$ such that

$$
\ell\left(\tilde{S} ; P_{d}\left(S_{B}\right)\right)=P_{i}(\tilde{S})
$$

Figure 13 shows that if $S_{T}<\tilde{S}$, then there exists a unique $u_{B}^{*} \in\left(P_{i}\left(S_{B}\right), P_{d}\left(S_{B}\right)\right)$ for which the following holds: All orbits starting at $\left(S_{B}, u=u_{B} \geq u_{B}^{*}\right)$ remain in $\overline{\mathcal{H}}$ and follow the corresponding scanning curves until $\overline{E_{T, i} E_{T, d}}$ is reached. The underlying travelling waves are solutions of (3.7). They result in monotone saturation and pressure profiles for any $\tau>0$. Orbits starting at $\left(S_{B}, u_{B}<u_{B}^{*}\right)$ intersect $\check{u}$ at some $S<S_{T}$ and enter the region $\check{\mathcal{H}}$. When $S_{T}>\tilde{S}$ any orbit leaving from $\overline{E_{B, i} E_{B, d}}$ intersects $\check{u}$ and enters the region $\mathcal{H}$. The saddle point $E_{B, i}$ needs special attention. If the orbit leaves $E_{B, i}$ into region $\check{\mathcal{H}}$, its local direction $\frac{d u}{d S}\left(S_{B}\right)$, see [14, Section 3], must satisfy

$$
\frac{d u}{d S}\left(S_{B}\right)=\frac{P_{i}^{\prime}\left(S_{B}\right)}{2}\left[1+\sqrt{1-\frac{4 c \tau \mathcal{G}^{\prime}\left(S_{B} ; S_{B}, S_{T}\right)}{\left(P_{i}^{\prime}\left(S_{B}\right)\right)^{2}}}\right] \leq \frac{d \check{u}}{d S}\left(S_{B}\right) .
$$

We have

Proposition 3.1. Condition (3.11b) at $S_{B} \Longleftrightarrow$ inequality (3.14).

Proof. We use the short hand notation $P_{i}^{\prime}:=P_{i}^{\prime}\left(S_{B}\right)$ and $\mathcal{G}^{\prime}=\mathcal{G}^{\prime}\left(S_{B} ; S_{B}, S_{T}\right)$. Evaluation of (3.11b) at $S_{B}$ gives

$$
1+\varepsilon P_{i}^{\prime}+\varepsilon^{2} c \tau \mathcal{G}^{\prime} \geq 0
$$


or $-\frac{1}{P_{i}^{\prime}} \geq \varepsilon+\frac{\varepsilon^{2} c \tau \mathcal{G}^{\prime}}{P_{i}^{\prime}}$. Hence, as $\mathcal{G}^{\prime}, P_{i}^{\prime}<0$ we get

$$
1-\frac{4 c \tau \mathcal{G}^{\prime}}{\left(P_{i}^{\prime}\right)^{2}} \geq 1+\frac{4 \varepsilon c \tau \mathcal{G}^{\prime}}{P_{i}^{\prime}}+\left(\frac{2 \varepsilon c \tau \mathcal{G}^{\prime}}{P_{i}^{\prime}}\right)^{2}=\left(1+\frac{2 \varepsilon c \tau \mathcal{G}^{\prime}}{P_{i}^{\prime}}\right)^{2}
$$

implying

$$
\sqrt{1-\frac{4 c \tau \mathcal{G}^{\prime}}{\left(P_{i}^{\prime}\right)^{2}}} \geq 1+\frac{2 \varepsilon c \tau \mathcal{G}^{\prime}}{P_{i}^{\prime}}
$$

This gives

$$
\frac{P_{i}^{\prime}}{2}+\frac{P_{i}^{\prime}}{2} \sqrt{1-\frac{4 c \tau \mathcal{G}^{\prime}}{\left(P_{i}^{\prime}\right)^{2}}} \leq P_{i}^{\prime}+c \varepsilon \tau \mathcal{G}^{\prime}
$$

which is inequality (3.14).

Summarizing the above gives the following picture. When conditions (3.11) are fulfilled, orbits that leave from points $E_{B}=\left(S_{B}, u_{B}\right) \in \overline{E_{B, i} E_{B, d}}$, with $u_{B}>P_{i}\left(S_{B}\right)$, either stay in $\overline{\mathcal{H}}$ and reach corresponding points of $\overline{E_{T, i} E_{T, d}}$ directly, or cross the lower bound $\check{u}$ and enter the region $\check{\mathcal{H}}$. The orbit that leaves $E_{B, i}$ enters directly $\check{\mathcal{H}}$. Once the orbits are in $\check{\mathcal{H}}$, we are in a situation that compares to Theorem 2.1 and Remark 2.2 since in $\check{\mathcal{H}}$ one has

$$
S^{\prime}=\frac{1}{c \tau}\left(P_{i}(S)-u\right)
$$

What remains is to show that any such orbit is captured by the segment $\overline{E_{T, i} E_{T, d}}$. We make this the content of the following theorem.

Theorem 3.1. Let $0<S_{B}<S_{T}<1, \tau>0$ and let conditions (3.11) and (3.1) be satisfied. Further, let $(S, u)$ be the solution orbit that originates from a point $E_{B}=$ $\left(S_{B}, u_{B}\right) \in \overline{E_{B, i} E_{B, d}}$, where $u_{B}<u_{B}^{*}$ if $S_{T} \leq \tilde{S}$ or $u_{B} \in\left[P_{i}\left(S_{B}\right), P_{d}\left(S_{B}\right)\right]$ if $S_{T}>\tilde{S}$ (see Figure 13). Then the following holds

(i) If $\tau>\tau_{c, d}$, the orbit exceeds $S_{T}$ and reaches a point $E_{T} \in \overline{E_{T, i} E_{T, d}}$. This happens after, at most, a finite number of rotations around $\overline{E_{T, i} E_{T, d}}$;

(ii) If $\tau_{c, i}<\tau \leq \tau_{c, d}$, then in addition, the orbit may reach the boundary point $E_{T, d}$ from $\hat{\mathcal{H}}$

(iii) If $\tau \leq \tau_{c, i}$, then in addition the orbit may reach the boundary point $E_{T, i}$ from $\check{\mathcal{H}}$. For small $\tau$ this may happen in a direct way, without exceeding $S_{T}$.

Proof. The proof has two parts. In the first we use again the divergence argument to demonstrate the existence of orbits connecting $E_{B}$ and corresponding points of $\overline{E_{T, i} E_{T, d}}$. In the second we show that the orbits can go around $\overline{E_{T, i} E_{T, d}}$ only finitely many times. 
To use the divergence argument we write $\vec{R}(\vec{u})$ as

$$
\vec{R}(\vec{u})=\left(\begin{array}{c}
\frac{1}{c \tau} \mathcal{E}(S, u) \\
\mathcal{G}\left(S ; S_{B}, S_{T}\right)
\end{array}\right)
$$

Hence

$$
\operatorname{div} \vec{R}=\frac{1}{c \tau} \frac{\partial \mathcal{E}}{\partial S} \text { with } \frac{\partial \mathcal{E}}{\partial S}= \begin{cases}P_{i}^{\prime}(S) & \text { when } u \in \hat{H}, \\ -c \tau \varepsilon \mathcal{G}^{\prime}\left(S ; S_{B}, S_{T}\right) & \text { when } u \in \mathcal{H}, \\ P_{d}^{\prime}(S) & \text { when } u \in \mathcal{H}_{d}\end{cases}
$$

Note that $\operatorname{div} \vec{R}$ does not have a fixed sign as the sign of $\mathcal{G}^{\prime}$ changes in $\overline{\mathcal{H}}$. With reference to Figure 11, we see that there are three possibilities for orbits that originate from $\overline{E_{B, i} E_{B, d}}$ :

Case (a) They go around $\overline{E_{T, i} E_{T, d}}$ and end up in $E_{B, d}$ or they leave the region $S>S_{B}$ through the segment $\left\{S=S_{B}, u>P_{d}\left(S_{B}\right)\right\}$.

Case (b) They go into a limit cycle around $\overline{E_{T, i} E_{T, d}}$.

Case (c) They approach the line $\overline{E_{T, i} E_{T, d}}$.

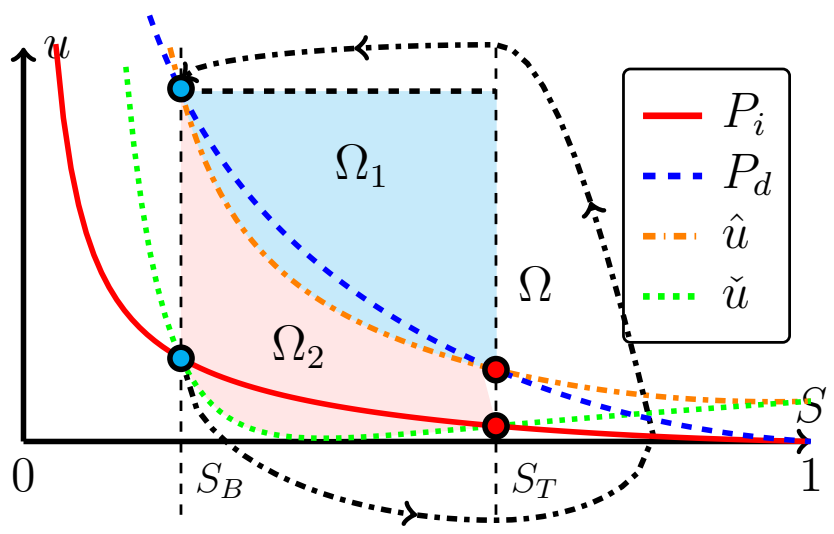

Figure 14: If Case (a) holds, then the orbit connects to the segment $\left\{S=S_{B}, u \geq P_{d}\left(S_{B}\right)\right\}$. The regions $\Omega, \Omega_{1}, \Omega_{2}$ used in the proof are marked by the colours.

We first consider the orbit leaving $E_{B, i}$. Case (a) for this orbit is shown in Figure 14. Let $\Omega$ be the region enclosed by the orbit and the line $S=S_{B}$. As shown in [14], the orbit can only exit through $S=S_{B}$ if

$$
\int_{\Omega} \operatorname{div} \vec{R}=\int_{\partial \Omega} \vec{u} \cdot \hat{n} \geq 0
$$


$\hat{n}$ being the unit vector that is outward normal to $\partial \Omega$. Define the regions $\Omega_{1}, \Omega_{2} \subset \Omega$ by

$$
\begin{aligned}
& \Omega_{1}=\left\{(S, u): S_{B}<S<S_{T}, \hat{u}<u<P_{d}\left(S_{B}\right)\right\}, \\
& \Omega_{2}=\left\{(S, u): S_{B}<S<S_{T}, \check{u}(S)<u<\hat{u}(S)\right\},
\end{aligned}
$$

and observe that

$$
\int_{\Omega /\left(\Omega_{1} \cup \Omega_{2}\right)} \operatorname{div} \vec{R} \leq 0
$$

since $\frac{\partial \mathcal{E}}{\partial S} \leq 0$ in $\Omega / \Omega_{2}$. Further note that

$$
\begin{aligned}
& \int_{\Omega_{1}} \operatorname{div} \vec{R}=\int_{S_{B}}^{S_{T}} \int_{\hat{u}(S)}^{P_{d}\left(S_{B}\right)} \frac{P_{d}^{\prime}(S)}{c \tau} d u d S=\frac{1}{c \tau} \int_{S_{B}}^{S_{T}} P_{d}^{\prime}(S)\left(P_{d}\left(S_{B}\right)-P_{d}(S)-c \tau \varepsilon \mathcal{G}(S)\right) d S \\
& =-\frac{1}{2 c \tau}\left(P_{d}\left(S_{T}\right)-P_{d}\left(S_{B}\right)\right)^{2}-\varepsilon \int_{S_{B}}^{S_{T}} P_{d}^{\prime} \mathcal{G} d S,
\end{aligned}
$$

and

$$
\begin{aligned}
& \int_{\Omega_{2}} \operatorname{div} \vec{R}=\int_{S_{B}}^{S_{T}} \int_{P_{i}(S)}^{P_{d}(S)}\left(-\varepsilon \mathcal{G}^{\prime}\right) d u d S=-\varepsilon \int_{S_{B}}^{S_{T}} \mathcal{G}^{\prime}\left(P_{d}(S)-P_{i}(S)\right) d S \\
& =-\left.\varepsilon \mathcal{G}\left(S ; S_{B}, S_{T}\right)\left(P_{d}(S)-P_{i}(S)\right)\right|_{S_{B}} ^{S_{T}}+\varepsilon \int_{S_{B}}^{S_{T}}\left(P_{d}^{\prime}-P_{i}^{\prime}\right) \mathcal{G} d S \\
& =\varepsilon \int_{S_{B}}^{S_{T}}\left(P_{d}^{\prime}-P_{i}^{\prime}\right) \mathcal{G} d S .
\end{aligned}
$$

Summing up gives

$$
\int_{\Omega} \operatorname{div} \vec{R}<-\frac{1}{2 c \tau}\left(P_{d}\left(S_{T}\right)-P_{d}\left(S_{B}\right)\right)^{2}-\varepsilon \int_{S_{B}}^{S_{T}} P_{i}^{\prime} \mathcal{G} d S<0 .
$$

This contradicts (3.16) and hence the orbit cannot exit through the segment $\left\{S=S_{B}, u \geq\right.$ $\left.P_{d}\left(S_{B}\right)\right\}$. As no two orbits can intersect, the orbit originating from any point on $\overline{E_{B, i} E_{B, d}}$ cannot leave the region $\left\{S>S_{B}\right\}$. 


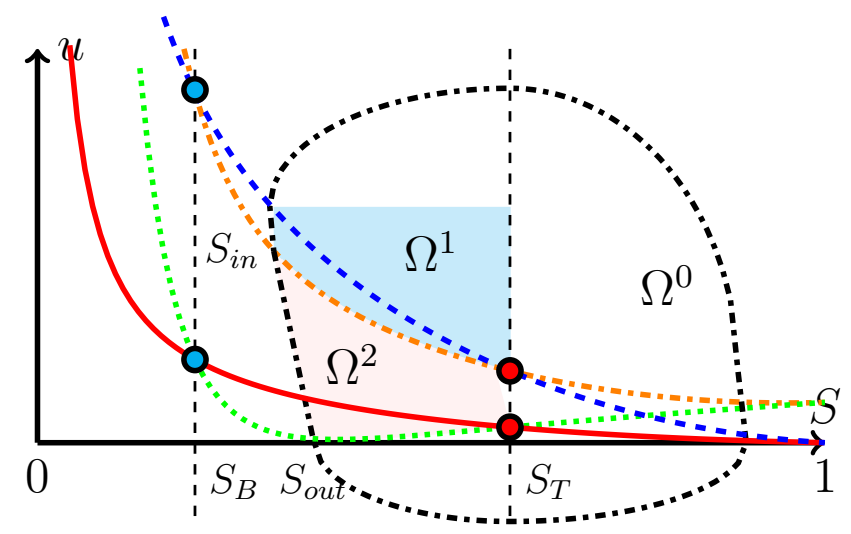

Figure 15: Case (b): If the orbits go to a limit cycle then the limit cycle will be as the black dashdotted closed loop. The regions $\Omega^{0}, \Omega^{1}, \Omega^{2}$ and $\left(S_{\text {in }}, S_{\text {out }}\right)$ are used in the proof.

We need to further eliminate Case (b). As can be seen from Figure 13 a limit cycle clearly cannot exist for $S_{T} \leq \tilde{S}$. For $S_{T}>\tilde{S}$, if the limit cycle exists, then it will be as shown in Figure 15. Let $\Omega^{0}$ be the region bounded by the closed loop and let $\left(S_{\text {in }}, S_{\text {out }}\right)$ be saturation values at which the limit cycle enters and leaves the region $\overline{\mathcal{H}}$ such that $S_{\text {in }}<S_{\text {out }}<S_{T}$. Further, let $\Omega^{1}:=\left\{(S, u) \in \Omega^{0}: S_{\text {in }}<S<S_{T}, \hat{u}(S)<u<P_{d}\left(S_{\text {in }}\right)\right\}$ and let $\Omega^{2} \subset \Omega^{0}$ be the open subregion in $S<S_{T}$ bounded by $S=S_{T}, u=\hat{u}(S)$, $u=\breve{u}(S)$ and the closed loop. Again, we have $\operatorname{div} \vec{R}<0$ in $\Omega^{0} / \Omega^{2}$. The integral of $\operatorname{div} \vec{R}$ over $\Omega^{1}$ is

$$
\begin{aligned}
& \int_{\Omega^{1}} \operatorname{div} \vec{R}=\frac{1}{c \tau} \int_{S_{i n}}^{S_{T}} P_{d}^{\prime}(S)\left(P_{d}\left(S_{i n}\right)-P_{d}(S)-c \tau \varepsilon \mathcal{G}(S)\right) d S \\
& =-\frac{1}{2 c \tau}\left(P_{d}\left(S_{T}\right)-P_{d}\left(S_{i n}\right)\right)^{2}-\varepsilon \int_{S_{i n}}^{S_{T}} P_{d}^{\prime} \mathcal{G} d S
\end{aligned}
$$

and over $\Omega^{2}$ is

$$
\int_{\Omega^{2}} \operatorname{div} \vec{R}=-\varepsilon \int_{S_{\text {in }}}^{S_{\text {out }}} \mathcal{G}^{\prime}\left(\int_{P_{d}\left(S_{\text {in }}\right)-\frac{1}{\varepsilon}\left(S-S_{\text {in }}\right)}^{P_{d}(S)+c \tau \mathcal{G}} d u\right) d S-\varepsilon \int_{S_{\text {out }}}^{S_{T}} \mathcal{G}^{\prime}\left(\int_{\tilde{u}(S)}^{\hat{u}(S)} d u\right) d S .
$$

Integrating by parts yields

$$
\begin{aligned}
& \int_{\Omega^{2}} \operatorname{div} \vec{R}=\varepsilon \int_{S_{\text {in }}}^{S_{T}} P_{d}^{\prime} \mathcal{G}+\int_{S_{\text {in }}}^{S_{\text {out }}}\left(1+c \tau \varepsilon^{2} \mathcal{G}^{\prime}\right) \mathcal{G}-\int_{S_{\text {out }}}^{S_{T}} P_{i}^{\prime} \mathcal{G} \\
& \leq \varepsilon \int_{S_{\text {in }}}^{S_{T}} P_{d}^{\prime} \mathcal{G}-\int_{S_{\text {in }}}^{S_{\text {out }}} P_{i}^{\prime} \mathcal{G}-\int_{S_{\text {out }}}^{S_{T}} P_{i}^{\prime} \mathcal{G}=\varepsilon \int_{S_{\text {in }}}^{S_{T}} P_{d}^{\prime} \mathcal{G}-\varepsilon \int_{S_{\text {in }}}^{S_{T}} P_{i}^{\prime} \mathcal{G} .
\end{aligned}
$$

In the last step, condition (3.11b) was used. Summing up the integrals gives

$$
\int_{\Omega^{0}} \operatorname{div} \vec{R}<-\frac{1}{2 c \tau}\left(P_{d}\left(S_{T}\right)-P_{d}\left(S_{i n}\right)\right)^{2}-\varepsilon \int_{S_{i n}}^{S_{T}} P_{i}^{\prime} \mathcal{G} d S<0 .
$$


As before, this gives a contradiction. Hence we are left with Case (c).

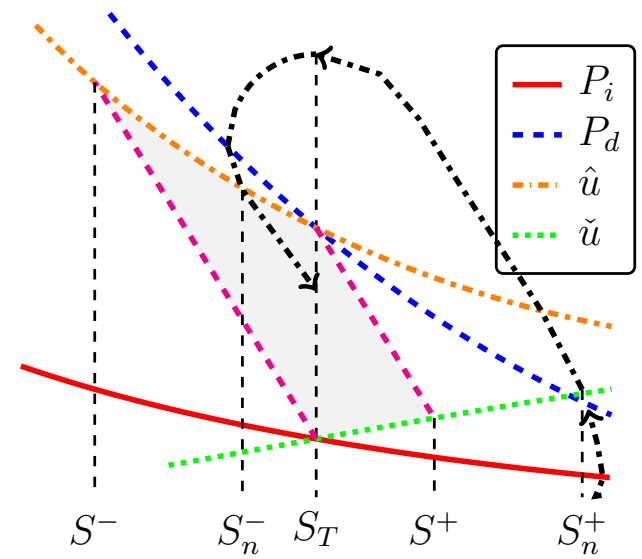

Figure 16: The points $S^{ \pm}$and $S_{n}^{ \pm}$and the final turn of the orbit.

What remains is to show that the orbit goes at most finitely many times around $\overline{E_{T, i} E_{T, d}}$ before it reaches one of its points. Define, see Figure 16, $S^{+}>S_{T}$ and $S^{-}<S_{T}$ such that

$$
\hat{u}\left(S^{-}\right)+\frac{1}{\varepsilon} S^{-}=P_{i}\left(S_{T}\right)+\frac{1}{\varepsilon} S_{T} \text { and } \check{u}\left(S^{+}\right)+\frac{1}{\varepsilon} S^{+}=P_{d}\left(S_{T}\right)+\frac{1}{\varepsilon} S_{T},
$$

If the orbit enters $\overline{\mathcal{H}}$ in the $n^{\text {th }}$ turn at $S=S_{n}^{-}$with $S_{n}^{-} \in\left(S^{-}, S_{T}\right)$, then this is the final turn of the orbit. Similarly, if the orbit enters $\overline{\mathcal{H}}$ at the $n^{\text {th }}$ turn at $S=S_{n}^{+}$with $S_{n}^{+} \in\left(S_{T}, S^{+}\right)$, then this is the final turn. If no such $n$ exists, then the orbit must go into a limit cycle whose closed loop contains the shaded region in Figure 16. This clearly gives a contradiction.

Statements (ii) and (iii) of Theorem 3.1 follow from the nature of the eigenvalues, see also the proof of Lemma 2.2 .

Conditions (3.11) are sufficient but not necessary to prove existence as described in Theorem 3.1. Below we give an (almost) explicit construction of a solution when (3.11) is violated.

Example 3.1 Let $S_{B} \in(0,1)$ and $\varepsilon>0$ be given, and let $P_{i}$ and $P_{d}, S_{T} \in\left(S_{B}, 1\right)$ and $u_{B}^{*}$ be such that $\ell\left(S ; u_{B}\right)$, given by $(3.8)$, satisfies

$$
\ell\left(S_{T}, u_{B}^{*}\right)=P_{i}\left(S_{T}\right)
$$

see Figure 11 and Figure 17. 


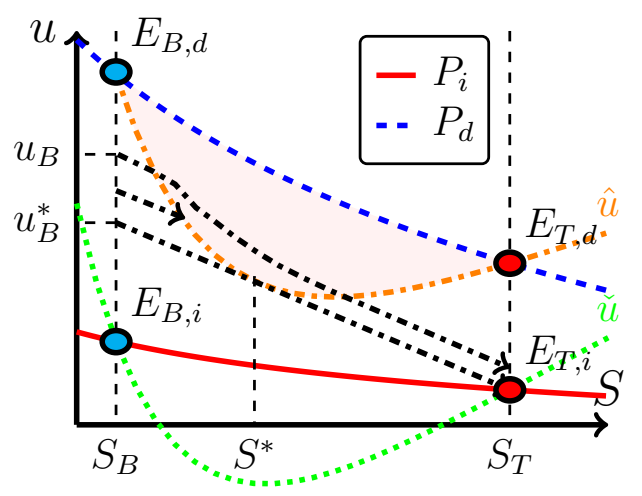

Figure 17: Construction of orbit when (3.11) is violated. Here the function $\hat{u}$ is convex in $\left(S_{B}, S_{T}\right)$ and for $\tau=\tau^{*}$ it touches $\ell\left(S, u^{*}\right)$ at $S=S^{*}$.

Further assume $P_{d} \in C^{2}(0,1)$ and $P_{d}^{\prime \prime}(S)>0$ for $S_{B}<S<S_{T}$. For the relative permeability we consider the explicit case $k(S)=S^{2}$. A straightforward calculation gives from $(2.8)$ that $\mathcal{G}$ satisfies

$$
\mathcal{G}^{\prime \prime}>0 \text { in }\left[S_{B}, S_{T}\right]
$$

provided $S_{T}<2 S_{B}$. As a consequence,

$$
\hat{u}^{\prime \prime}=P_{d}^{\prime \prime}+c \varepsilon \tau \mathcal{G}^{\prime \prime}>0 \text { in }\left(S_{B}, S_{T}\right),
$$

if $S_{T}<2 S_{B}$. Next we increase $\tau$ from $\tau=0$, when $\hat{u}=P_{d}$, until $\tau=\tau^{*}$ when $\hat{u}$ touches the line $\ell\left(S ; u_{B}^{*}\right)$ at some $S=S^{*}$. By the convexity we have

$$
\hat{u}^{\prime}<-\frac{1}{\varepsilon} \text { for } S_{B} \leq S<S^{*} \text {. }
$$

The monotonicity of the orbits in the shaded region in Figure 17 ensures the existence of orbit running from $E_{B}=\left(S_{B}, u_{B}\right)$, with $u_{B} \in\left[u_{B}^{*}, P_{d}\left(S_{B}\right)\right]$, to a corresponding point $E_{T} \in \overline{E_{T, i} E_{T, d}}$. The saturation and pressure profiles are monotone in this example.

If $(3.11 \mathrm{~b})$ is satisfied, the orbit from $E_{B, i}$ directly enters $\check{\mathcal{H}}$, and does not feel the influence of the parameter $\varepsilon$. Hence, for this orbit, the condition of whether it remains to the left of $S=1$ is the same as for play-type hysteresis, see Theorem 2.1. Moreover, as the orbits are ordered and cannot intersect when $S<1$, the same applies to any orbit leaving $\overline{E_{B, i} E_{B, d}}$. Only if $S_{T}>S_{T}^{*}\left(S_{B}\right)$ (or $\alpha\left(S_{B}, S_{T}\right)<0$ ) and $\tau$ is sufficiently large orbits can reach $S=1$. Here one has to be careful with condition (3.11) which requires a certain smallness of the product $\varepsilon \tau^{2}$. Large values of $\tau$ are allowed provided $\varepsilon$ is sufficiently small.

\section{Numerical results}

In this section we discuss a number of numerical PDE-solutions and compare the results to the theoretical travelling wave results. Clearly, in the numerical approach one works 
in a truncated domain: $-W<x<W$ for some $W>0$, chosen sufficiently large so that well-developed saturation profiles appear.

For the play-type model, the equations are (1.1) and (1.5), where we write the latter as $\partial_{t} S=\frac{1}{\tau f(S)} \mathcal{F}(S, u)$, see expression (2.7). Combining the equations and using the semi-implicit time discretization scheme described in [14], we have

$$
\begin{aligned}
& S_{n}=S_{n-1}+\frac{\Delta t}{\tau f(S)} \mathcal{F}\left(S_{n-1}, u_{n-1}\right), \\
& \frac{1}{\tau f\left(S_{n}\right)} \mathcal{F}\left(S_{n}, u_{n}\right)+\partial_{x}\left(k\left(S_{n}\right)\left(\partial_{x} u_{n}+1\right)\right)=0 .
\end{aligned}
$$

Here $\Delta t>0$ is the time step and $n \in\{1, . ., N\}$ for some $N \in \mathbb{N}$. The pair $\left(S_{n}, u_{n}\right)$ approximates $(S, u)$ at the $n^{\text {th }}$ time step, i.e. at $t=n \Delta t$. The initial condition for the saturation at $n=0$ is a smooth approximation of the Riemann data

$$
S_{0}:= \begin{cases}S_{T} & \text { if } x<0 \\ S_{B} & \text { if } x>0\end{cases}
$$

The boundary conditions are

$$
\begin{array}{lr}
k\left(S_{n}\right)\left(\partial_{x} u_{n}+1\right)=k\left(S_{T}\right) & \text { at } x=-W, \\
u_{n}=u_{B} \in\left[P_{i}\left(S_{B}\right), P_{d}\left(S_{B}\right)\right] & \text { at } x=W,
\end{array}
$$

for each $n \geq 1$. The first boundary condition implies that we prescribe a constant water flux $k\left(S_{T}\right)$ at $x=-W$. For well-developed profiles this means that approximately $S_{n}(-W)=S_{T}$ for $n$ sufficiently large. As for the second condition, we will see that independent of the choice of $u_{B}$, the profile develops into a travelling wave as predicted. Taking $u_{B}=P_{i}\left(S_{B}\right)$ gives a compatible boundary condition. Taking any $u_{B} \neq P_{i}\left(S_{B}\right)$, gives a boundary layer at $x=W$.

For the extended model, one needs to discretize the combination (1.1) and (1.7) in the domain $(-W, W)$. Here we propose the semi-implicit scheme

$$
\begin{aligned}
& S_{n}=S_{n-1}+\Delta t \partial_{x}\left(k\left(S_{n-1}\right)\left(\partial_{x} u_{n-1}+1\right)\right), \\
& \mathcal{A}\left(S_{n}, u_{n}, \frac{u_{n}-u_{n-1}}{\Delta t}\right)+\partial_{x}\left(k\left(S_{n}\right)\left(\partial_{x} u_{n}+1\right)\right)=0,
\end{aligned}
$$

for $n \geq 1$. The function $\mathcal{A}:(0,1) \times \mathbb{R}^{2} \rightarrow \mathbb{R}$ is defined in (1.7). Initial and boundary conditions are again given by (4.2) and (4.3). To solve equations (4.4), an initial condition for the pressure is required as well. In the computations we have taken

$$
u(x, 0)=\frac{1}{2}\left(P_{i}(S(x, 0))+P_{d}(S(x, 0))\right) \text { for }-W<x<W .
$$


To avoid a boundary layer at $x=W$, we take in $(4.3 \mathrm{~b})$

$$
u_{B}=\frac{1}{2}\left(P_{i}\left(S_{B}\right)+P_{d}\left(S_{B}\right)\right)
$$

Note that for $x<0$, the initial condition need not be compatible with the unique pressure $u_{T} \in \overline{E_{T, i} E_{T, d}}$ that results from the traveling wave analysis with $u_{B}$ given by (4.5b). This pressure is approximately attained after a sufficiently large number of time steps.

Distinguishing the cases $\frac{\varepsilon \tau f(S)}{\Delta t} \lessgtr 1$, one straightforwardly verifies that $\mathcal{A}\left(S_{n}, u_{n}, u_{n}-\right.$ $\left.u_{n-1} / \Delta t\right)$ strictly decreases with respect to $u_{n}$. Hence, for given $S_{n}, u_{n-1} \in L^{2}((-W, W))$, such that $k\left(S_{n}\right) \geq \delta$ for some $\delta>0$, there exists a unique $u_{n} \in H^{1}(-W, W)$ satisfying (4.4b) and (4.3), e.g. see [16, Chapter 2]. Equations (4.1b) and (4.4b) are solved numerically by means of the L-scheme $[10,14]$.

Finite differences are used to solve equations (4.1) and (4.4), with $\Delta x=0.2$ and $\Delta t=0.02$. The length of the domain $(2 W)$ is chosen according to the problem. The nonlinearities $k, P_{i}$ and $P_{d}$ are as in [6] and we restrict ourselves to $f(S)=1$ only. The extension of pressures $P_{k}(k=i, d)$ is achieved by setting $P_{k}=10^{3} \cdot(1-S)$ for $S>1$.
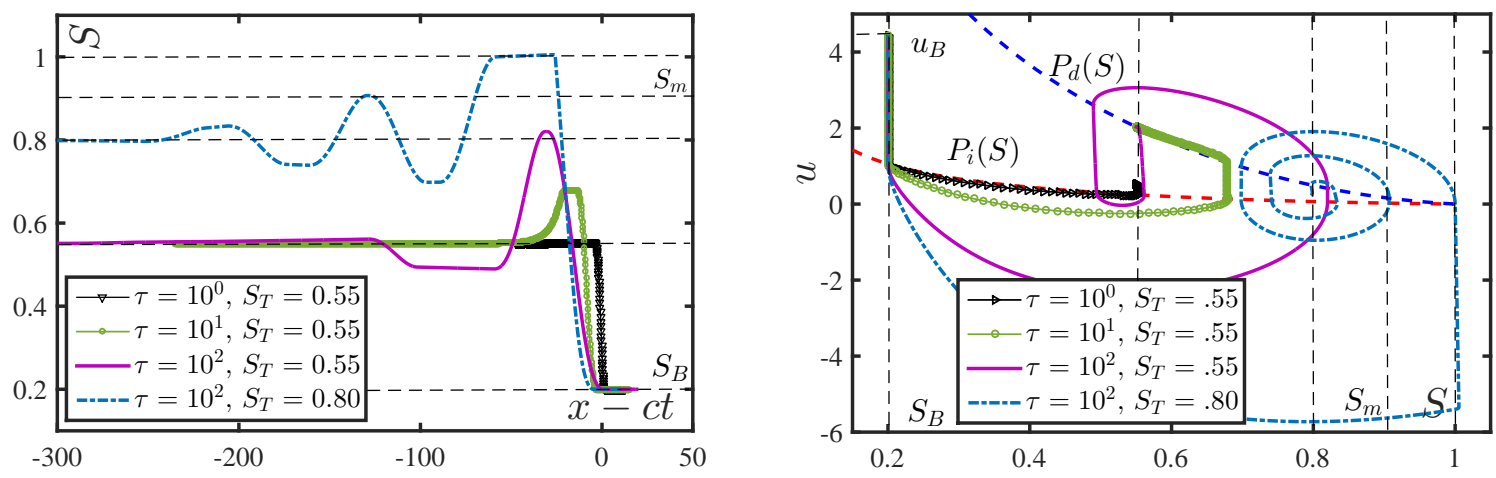

Figure 18: Play-type hysteresis: (left) $S$ vs. $x-c t$ plots and (right) $u$ vs. $S$ plots for various $S_{T}$ and $\tau$ values with $S_{B}=0.2$ fixed. Here $c$ is calculated from $S_{B}$ and $S_{T}$ using (2.5). For $S_{B}=0.2$ it follows that $S_{T}^{*}\left(S_{B}\right) \approx 0.6$, see Figure 4 . Results for $S_{T}=0.55<S_{T}^{*}\left(S_{B}\right)$ are shown first. Corresponding value of $S_{m}$ is $S_{m} \approx 0.9$. Profiles for three $\tau$ values are plotted: $\tau=1<\tau_{c, i}, \tau=10 \in\left[\tau_{c, i}, \tau_{c, d}\right]$ and $\tau=100>\tau_{c, d}$. The behaviour is as expected and the saturation lies below $S_{m}<1$. Also the result for $S_{T}=0.8>S_{T}^{*}\left(S_{B}\right)$ and $\tau=100>\tau_{c, d}$ is shown. In this case the satuartion reaches the maximum value and a plateau can be seen at $S=1$.

The results for play-type hysteresis are shown in Figure 18. They agree well with the predictions. Fronts quickly develop into travelling waves with wave-speed $c$ predicted by (2.5). For $S_{T}<S_{T}^{*}\left(S_{B}\right)$ the profiles stay away from $S=1$ and the behaviour with respect to $\tau$ is as to be expected. For $\tau<\tau_{c, i}$ the travelling wave orbit goes directly to $E_{T, i}$; 
for $\tau \in\left[\tau_{c, i}, \tau_{c, d}\right)$ it goes around $E_{T, i}$ to reach $E_{T, d}$ and for $\tau>\tau_{c, d}$ it oscillates around $\overline{E_{T, i} E_{T, d}}$, though infinitely many oscillations cannot be seen due to the finite domain size. For $S_{T}>S_{T}^{*}\left(S_{B}\right)$ and $\tau$ large enough, the profile indeed reaches $S=1$ and a plateau is observed.

Figure 19 shows results for extended play-type hysteresis. The scanning curves within the hysteretic region are inclined by the fixed angle $\tan ^{-1}\left(-\frac{1}{\varepsilon}\right)$, where $\varepsilon=\frac{1}{40}$ is used. Observe that, for all the $(\varepsilon, \tau)$ pairs used in Figure 19, conditions (3.11) are satisfied. In all cases, small irregularities are observed at the locations where orbits meet the segment $\overline{E_{T, i} E_{T, d}}$. This reflects the behaviour in $\{x<0\}$ as observed earlier in this section. These irregularities disappear when $n$ becomes large.
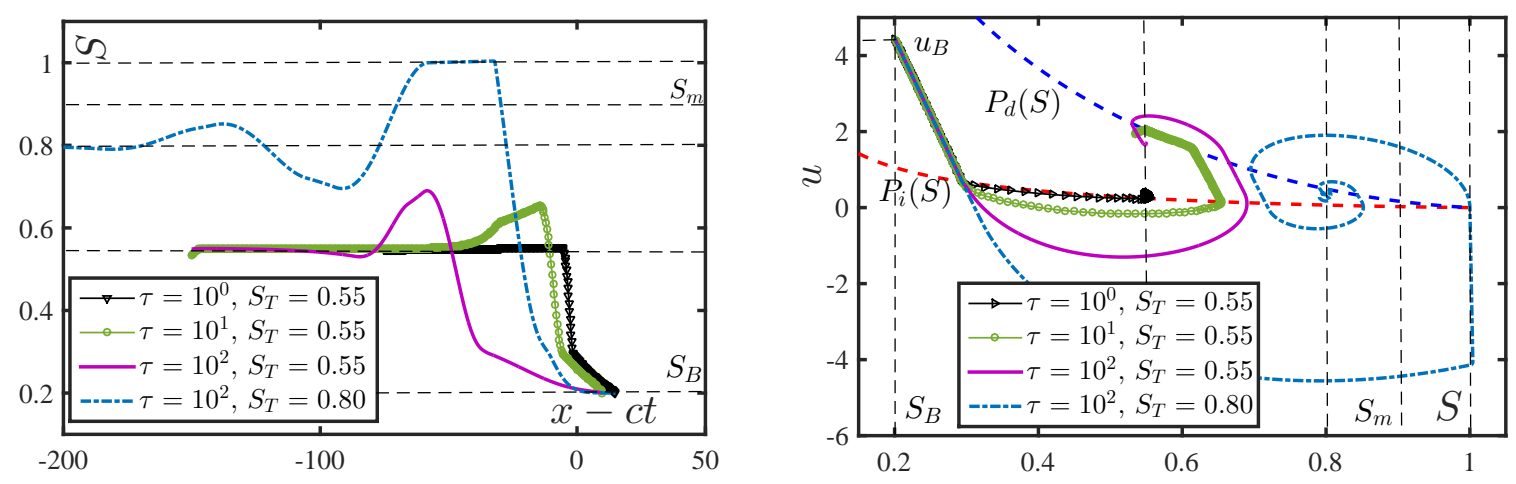

Figure 19: Extended play-type hysteresis: (left) $S$ vs. $x-c t$ plots and (right) $u$ vs. $S$ plots for various $S_{T}$ and $\tau$ values with $S_{B}=0.2$ and $\varepsilon=\frac{1}{40}$ fixed. Fronts develop into travelling wave profiles with wave-speed $c$ given by $(2.5)$. Here also, $S_{T}^{*}\left(S_{B}\right) \approx 0.6$. As before, results for $S_{T}=0.55<S_{T}^{*}\left(S_{B}\right)$ are shown first for three different $\tau$ values: $\tau=1<\tau_{c, i}, \tau=10 \in\left[\tau_{c, i}, \tau_{c, d}\right]$ and $\tau=100>\tau_{c, d}$. The orbits have a constant slope of $-\frac{1}{\varepsilon}$ in the hysteretic region $\mathcal{\mathcal { H }}$ and are bounded above by $S_{m}<1$. Finally, the result for $S_{T}=0.8>S_{T}^{*}\left(S_{B}\right)$ and $\tau=100>\tau_{c, d}$ is shown. For all the plots, $u_{B}$ is as in (4.5b).

\section{Conclusion}

In this paper we studied the behaviour of infiltration fronts in a long homogeneous vertical unsaturated porous column. The main focus was on describing the fronts when nonequilibrium effects, such as capillary hysteresis and dynamic capillarity are considered. For this purpose, travelling wave approximation was used. Two models for hysteresis were considered, the play-type hysteresis and the extended play-type hysteresis model.

In case of play-type hysteresis model, it was shown that for small dynamic capillary constant $\tau$, the fronts will be monotone. If $\tau$ is larger than thresholds calculated from known parameters, the fronts will first develop finitely many and then infinitely many overshoots. Moreover, saturation plateaus will form at the location of undershoots and 
overshoots and the plateaus will become wider as one moves away from the wave-front. Criteria for the saturation overshoots to reach full saturation was also investigated. If the integral (2.27), which depends solely on the parameters of the system, is positive then the saturation cannot reach the maximum possible saturation value even if $\tau$ is large. Similarly, if $f \notin L^{1}(0,1)$, then the saturation cannot reach the maximum value.

To explain in more detail behaviour of wetting fronts observed from experiments, we also considered extended play-type hysteresis model which incorporates non-vertical scanning curves. The existence of travelling wave solutions were proved under conditions (3.11). One of the key differences in this case is that, if the initial saturation and the injection saturation are close enough then there will be no overshoots irrespective of the value of $\tau$. Another important distinction is that, in this case only finitely many oscillations can occur. This is also observed from experiments $[2,5]$.

Finally, a set of numerical tests were performed showing that the fronts indeed develop into travelling wave profiles for long columns. The predictions from our analysis were reproduced by the numerical results.

\section{Acknowledgements}

C.J. van Duijn acknowledges the support of the Darcy Centre of Utrecht University and Eindhoven University of Technology. K. Mitra is supported by Shell and the Netherlands Organisation for Scientific Research (NWO) through the CSER programme (project 14CSER016) and by the Hasselt University through the project BOF17BL04.

\section{References}

[1] A.Y. Beliaev and S.M. Hassanizadeh. A theoretical model of hysteresis and dynamic effects in the capillary relation for two-phase flow in porous media. Transport in Porous Media, 43(3):487-510, 2001.

[2] S. Bottero, S.M. Hassanizadeh, P.J. Kleingeld, and T.J. Heimovaara. Nonequilibrium capillarity effects in two-phase flow through porous media at different scales. Water Resources Research, 47(10), 2011.

[3] G. Camps-Roach, D.M. O'Carroll, T.A. Newson, T. Sakaki, and T.H. Illangasekare. Experimental investigation of dynamic effects in capillary pressure: Grain size dependency and upscaling. Water Resources Research, 46(8), 2010.

[4] C. Cuesta, C.J. van Duijn, and J. Hulshof. Infiltration in porous media with dynamic capillary pressure: travelling waves. European Journal of Applied Mathematics, 11(4):381-397, 2000. 
[5] D.A. DiCarlo. Experimental measurements of saturation overshoot on infiltration. Water Resources Research, 40(4), 2004.

[6] C.J. van Duijn and K. Mitra. Hysteresis and horizontal redistribution in porous media. Transport in Porous Media, 122(2):375-399, Mar 2018.

[7] J. Koch, A. Rätz, and B. Schweizer. Two-phase flow equations with a dynamic capillary pressure. European Journal of Applied Mathematics, 24(1):49-75, 2013.

[8] N.R. Morrow and C.C. Harris. Capillary equilibrium in porous materials. Society of Petroleum Engineers, 1965.

[9] J.L. Nieber, R.Z. Dautov, A.G. Egorov, and A.Y. Sheshukov. Dynamic capillary pressure mechanism for instability in gravity-driven flows; review and extension to very dry conditions. Transport in Porous Media, 58(1-2):147-172, 2005.

[10] I.S. Pop, F.A. Radu, and P. Knabner. Mixed finite elements for the Richards equation: linearization procedure. Journal of Computational and Applied Mathematics, 168(1):365-373, 2004.

[11] A. Rätz and B. Schweizer. Hysteresis models and gravity fingering in porous media. ZAMM-Journal of Applied Mathematics and Mechanics/Zeitschrift für Angewandte Mathematik und Mechanik, 94(7-8):645-654, 2014.

[12] G.C. Sander, O.J. Glidewell, and J. Norbury. Dynamic capillary pressure, hysteresis and gravity-driven fingering in porous media. In Journal of Physics: Conference Series, volume 138, page 012023. IOP Publishing, 2008.

[13] C.J. van Duijn, Y. Fan, L.A. Peletier, and I.S. Pop. Travelling wave solutions for degenerate pseudo-parabolic equations modelling two-phase flow in porous media. Nonlinear Analysis: Real World Applications, 14(3):1361-1383, 2013.

[14] C.J. van Duijn, K. Mitra, and I.S. Pop. Travelling wave solutions for the Richards equation incorporating non-equilibrium effects in the capillarity pressure. Nonlinear Analysis: Real World Applications, 41(Supplement C):232 - 268, 2018.

[15] A. Visintin. Differential models of hysteresis. Springer Science \& Business Media, 2013.

[16] E. Zeidler. Applied Functional Analysis: Applications to Mathematical Physics (Applied Mathematical Sciences)(v. 108). Springer, 1995.

[17] H. Zhang and P.A. Zegeling. A numerical study of two-phase flow models with dynamic capillary pressure and hysteresis. Transport in Porous Media, 116(2):825846, Jan 2017. 


\section{Appendix A Extended play-type hysteresis: non-vertical scanning curves}

We write the pressure expression (1.6) as

$$
u \in P^{+}(S)-P^{-}(S) \operatorname{sign}\left(\partial_{t} S+\varepsilon \partial_{t} u\right)-\tau f(S) \partial_{t} S,
$$

where

$$
P^{+}(S)=\frac{P_{d}(S)+P_{i}(S)}{2}, \quad P^{-}(S)=\frac{P_{d}(S)-P_{i}(S)}{2} .
$$

This form can be rewritten as

$$
\frac{\tau f(S)}{P^{-}(S)} \partial_{t}(S+\varepsilon u)+\operatorname{sign}\left(\partial_{t}(S+\varepsilon u)\right) \ni \frac{P^{+}(S)-u}{P^{-}(S)}+\frac{\varepsilon \tau f(S)}{P^{-}(S)} \partial_{t} u .
$$

Introducing the function, for each $0<S<1$,

$$
\Phi_{s}(r)= \begin{cases}\frac{P^{-}(S)}{\tau f(S)}(r-1) & \text { for } r \geq 1 \\ 0 & \text { for }-1<r<1 \\ \frac{P^{-}(S)}{\tau f(S)}(r+1) & \text { for } r \leq-1\end{cases}
$$

we deduce from (A.3)

$$
\partial_{t}(S+\varepsilon u)=\Phi_{s}\left(\frac{P^{+}(S)-u}{P^{-}(S)}+\frac{\varepsilon \tau f(S)}{P^{-}(S)} \partial_{t} u\right) .
$$

Evaluating the different cases in (A.4) and using the definitions from (A.2) gives

$$
\partial_{t} S= \begin{cases}\frac{P_{d}(S)-u}{\tau f(S)} & \text { when } u \geq P_{d}(S)+\varepsilon \tau f(S) \partial_{t} u \\ -\varepsilon \partial_{t} u & \text { when } P_{i}(S)+\varepsilon \tau f(S) \partial_{t} u<u<P_{d}(S)+\varepsilon \tau f(S) \partial_{t} u \\ \frac{P_{i}(S)-u}{\tau f(S)} & \text { when } u \leq P_{i}(S)+\varepsilon \tau f(S) \partial_{t} u\end{cases}
$$




\section{UHASSELT}

UHasselt Computational Mathematics Preprint Series

\section{8}

UP-18-03 Koondanibha Mitra and Hans van Duijn, Wetting fronts in unsaturated porous media: the combined case of hysteresis and dynamic capillary, 2018

UP-18-02 Xiulei Cao, Koondanibha Mitra, Error estimates for a mixed finite element discretization of a two-phase porous media flow model with dynamic capillarity, 2018

UP-18-01 Klaus Kaiser, Jonas Zeifang, Jochen Schütz, Andrea Beck and ClausDieter Munz, Comparison of different splitting techniques for the isentropic Euler equations, 2018

\section{7}

UP-17-12 Carina Bringedal, Tor Eldevik, Øystein Skagseth and Michael A. Spall, Structure and forcing of observed exchanges across the Greenland-Scotland Ridge, 2017

UP-17-11 Jakub Wiktor Both, Kundan Kumar, Jan Martin Nordbotten, Iuliu Sorin Pop and Florin Adrian Radu, Linear iterative schemes for doubly degenerate parabolic equations, 2017

UP-17-10 Carina Bringedal and Kundan Kumar, Effective behavior near clogging in upscaled equations for non-isothermal reactive porous media flow, 2017

UP-17-09 Alexander Jaust, Balthasar Reuter, Vadym Aizinger, Jochen Schütz and Peter Knabner, FESTUNG: A MATLAB / GNU Octave toolbox for the discontinuous Galerkin method. Part III: Hybridized discontinuous Galerkin (HDG) formulation, 2017 
UP-17-08 David Seus, Koondanibha Mitra, Iuliu Sorin Pop, Florin Adrian Radu and Christian Rohde, A linear domain decomposition method for partially saturated flow in porous media, 2017

UP-17-07 Klaus Kaiser and Jochen Schütz, Asymptotic Error Analysis of an IMEX Runge-Kutta method, 2017

UP-17-06 Hans van Duijn, Koondanibha Mitra and Iuliu Sorin Pop, Travelling wave solutions for the Richards equation incorporating non-equilibrium effects in the capillarity pressure, 2017

UP-17-05 Hans van Duijn and Koondanibha Mitra, Hysteresis and Horizontal Redistribution in Porous Media, 2017

UP-17-04 Jonas Zeifang, Klaus Kaiser, Andrea Beck, Jochen Schütz and ClausDieter Munz, Efficient high-order discontinuous Galerkin computations of low Mach number flows, 2017

UP-17-03 Maikel Bosschaert, Sebastiaan Janssens and Yuri Kuznetsov, Switching to nonhyperbolic cycles from codim-2 bifurcations of equilibria in DDEs, 2017

UP-17-02 Jochen Schütz, David C. Seal and Alexander Jaust, Implicit multiderivative collocation solvers for linear partial differential equations with discontinuous Galerkin spatial discretizations, 2017

UP-17-01 Alexander Jaust and Jochen Schütz, General linear methods for time-dependent PDEs, 2017

\section{6}

UP-16-06 Klaus Kaiser and Jochen Schütz, A high-order method for weakly compressible flows, 2016

UP-16-05 Stefan Karpinski, Iuliu Sorin Pop, Florin A. Radu, A hierarchical scale separation approach for the hybridized discontinuous Galerkin method, 2016

UP-16-04 Florin A. Radu, Kundan Kumar, Jan Martin Nordbotten, Iuliu Sorin Pop, Analysis of a linearization scheme for an interior penalty discontinuous Galerkin method for two phase flow in porous media with dynamic capillarity effects , 2016

UP-16-03 Sergey Alyaev, Eirik Keilegavlen, Jan Martin Nordbotten, Iuliu Sorin Pop, Fractal structures in freezing brine, 2016

UP-16-02 Klaus Kaiser, Jochen Schütz, Ruth Schöbel and Sebastian Noelle, A new stable splitting for the isentropic Euler equations, 2016 
UP-16-01 Jochen Schütz and Vadym Aizinger, A hierarchical scale separation approach for the hybridized discontinuous Galerkin method, 2016

All rights reserved. 